ACCEPTED/IN PRESS

DEVELOPMENTAL SCIENCE

(C) 2016 American Psychological Association. This paper is not the copy of record and may not exactly replicate the authoritative document published in the APA journal. Please do not copy or cite without authors permission.

The final article will be available, upon publication, at:

[DOI]

Accepted on 5/10/2017 


\title{
Children retain implicitly learned phonological sequences better than adults:
}

\author{
A longitudinal study \\ Eleonore H.M. Smalle ${ }^{1,2}$, Mike P.A. Page ${ }^{3}$, Wouter Duyck ${ }^{4}$, Martin Edwards ${ }^{1,2}$, Arnaud Szmalec ${ }^{1,2,4}$ \\ ${ }^{1}$ Psychological Sciences Research Institute, Université catholique de Louvain, Louvain-la-Neuve, \\ Belgium \\ ${ }^{2}$ Institute of Neuroscience, Université catholique de Louvain, Louvain-la-Neuve, Belgium \\ ${ }^{3}$ Department of Psychology, University of Hertfordshire, Hatfield, United Kingdom \\ ${ }^{4}$ Department of Experimental Psychology, Ghent University, Ghent, Belgium
}

Acknowledgement

This work was supported by a grant from the Fonds de la Recherche Scientifique - FRS - FNRS

(Belgium), grant "Crédit aux Chercheurs 1.A.915.14F," to the first author. The authors thank Prahlad Gupta and Steven Smith for making the TOTimals stimulus set available (Gupta, 2003; Schwartz \& Smith, 1997). The authors also thank all the teachers and pupils and, in particular, Dhr. Mallems, the school director of Sint-Bavo primary school (Ghent), for the time and cooperation they offered during data collection.

Correspondence Address

Eleonore Smalle

Université catholique de Louvain

Psychological Sciences Research Institute

Institute of Neuroscience

Place Cardinal Mercier 10, room C314

B-1348 Louvain-la-Neuve

E-mail: eleonore.smalle@uclouvain.be 


\section{Highlights}

- Children are very good at memorizing implicitly learnt word-forms

- Despite their higher short-term memory capacity, adults are more prone to forgetting

- The child advantage remains noticeable up to a consolidation period of one year

- The findings suggest developmental changes in implicit memory for novel word-forms 


\begin{abstract}
Whereas adults often rely on explicit memory, children appear to excel in implicit memory, which plays an important role in the acquisition of various cognitive skills, such as those involved in language. The current study aimed to test the assertion of an age-dependent shift in implicit versus explicit learning within a theoretical framework that explains the link between implicit sequence memory and word-form acquisition, using the Hebb repetition paradigm. We conducted a one-year, multiple-session longitudinal study in which we presented auditory sequences of syllables, copresented with pictures of aliens, for immediate serial recall by a group of children (8-9 years) and by an adult group. The repetition of one Hebb sequence was explicitly announced, while the repetition of another Hebb sequence was unannounced and, therefore, implicit. Despite their overall inferior recall performance, the children showed better offline retention of the implicit Hebb sequence, compared with adults who showed a significant decrement across the delays. Adults had gained more explicit knowledge of the implicit sequence than children, but this could not explain the age-dependent decline in the delayed memory for it. There was no significant age-effect for delayed memory of the explicit Hebb sequence, with both age groups showing retention. Overall performance by adults was positively correlated with measures of post-learning awareness. Performance by children was positively correlated with vocabulary knowledge. We conclude that children outperform adults in the retention over time of implicitly learned phonological sequences that will gradually consolidate into novel word-forms. The findings are discussed in the light of maturational differences for implicit versus explicit memory systems that also play a role in language acquisition.
\end{abstract}


Children appear to be superior in skill learning and in the acquisition of the implicit, procedural knowledge that underlies formal aspects of language (Johnson \& Newport, 1989; Newport, Bavelier, \& Neville, 2001; Ullman, 2004; Weber-Fox \& Neville, 1996). Adults, on the other hand, are argued to be superior on most tests of cognitive abilities, especially those that rely on explicit, declarative knowledge or attention (Craik \& Bialystok, 2006; Murphy, McKone, \& Slee, 2003). Recently, Janacsek and colleagues compared nine different age cohorts (from 4 to 85 years old) on the serial reaction time (SRT) task (Janacsek, Fiser, \& Nemeth, 2012). The SRT is a well-known implicit skill-learning task in which participants are asked to press a button that corresponds to a particular location on the screen. Unannounced to the participant, a particular sequence is repeated throughout the task, and results show better accuracy and faster response times for stimuli consistent with its sequence structure than for inconsistent stimuli. This is taken to indicate implicit learning of the sequence. Interestingly, the authors observed a peak in task performance before the age of twelve, after which performance dropped significantly. In another version of the task, however, where participants were explicitly made aware of the repetition, using instructions and visual cues, they found no age-dependent change in learning (Nemeth, Janacsek, \& Fiser, 2013). Nonetheless, in this latter case, additional measures of explicit knowledge concerning the repeating sequence (using verbal reports) revealed a different age-effect, with significantly better scores on explicit knowledge of the sequence after the age of twelve.

Based on these findings, Nemeth et al. (2013) argued that children's superiority in various skills might have its basis in underlying implicit-learning abilities that shift to explicit learning mechanisms later in life (Poldrack \& Packard, 2003). This might, in turn, be attributed to a neurological change at the level of two separate long-term memory systems, namely an early developed basal-ganglia system (the procedural memory system), that is involved in implicit skill learning, and the late maturing frontal/medial-temporal circuits (the declarative memory system), that is assumed to rely on explicit attention processes (Perez, Peynircioglu, \& Blaxton, 1998; Robertson, 2012; Squire, 1992). 
With respect to human language, only a few studies have directly compared implicit language-learning capacities between children and adults under equivalent learning conditions. Ferman and Karni (2010) for example, compared eight- and twelve-year old children and adults when learning the same implicit artificial-grammar rule, and found better within-session gains in adults. An interesting observation is, however, that children seem to have the capacity to outperform adults on the subsequent formation of long-term memory representations (i.e., in an offline consolidation phase), irrespective of sleep (Adi-Japha, Badir, Dorfberger, \& Karni, 2014; Ashtamker \& Karni, 2013; Ferman \& Karni, 2010; Wilhelm, Prehn-Kristensen, \& Born, 2012). In the study by Ferman and Karni (2010), reaction-time data revealed greater between-session gains, with respect to an implicitly learned grammar rule, for children than for adults, over a between-session delay of up to two months. Similarly, Bishop and colleagues found that children outperformed adults on the offline retention of novel word-forms (Bishop, Barry, \& Hardiman, 2012). This was found using a non-word repetition task in which unfamiliar polysyllabic nonwords were presented for repetition (session A), and represented for repetition after a delay of one hour (session B). Overall, the scores of the children tended to improve over the delay, while those of the adults declined significantly. No age differences were observed for learning within sessions, despite the observation that children initially started at a lower baseline, owing to the fact that they were exposed to the same learning material as adults. Importantly, the authors addressed the question of whether the decline between sessions observed in adults might be due to a ceiling effect at the end of the first session, allowing more room for them to decline across sessions compared with children. To answer this question, they compared children and adults who showed comparable scores on the last trial of session A. They found that these children still retained better across the one-hour delay than corresponding adults. This shows that children retain nonwords better than adults, even when initial learning performance is matched. This finding supports the hypothesis of a maturational effect in delayed memory which, as the authors argue, might have implications for the existence of sensitive periods in language-learning.

Here, we aimed to investigate child-adult differences in delayed memory for novel wordforms that were either acquired implicitly or explicitly through repetition learning of syllable 
sequences. We conducted a one-year, four-session learning study with a group of children (8-9 years old) and a group of adults, who were presented with novel sequences of nine syllables for immediate serial recall. These sequences were segmented into three chunks of three syllables by virtue of the copresentation of pictures of aliens, as described below. In the task we used, which is an adaptation of the well-known Hebb-learning task (Hebb, 1961), there were certain sequences of items (often called Hebb sequences) that were repeated in exactly the same order every third trial. Recall performance for such Hebb sequences usually improves relative to other non-repeating (filler) sequences. This finding, known as Hebb Repetition Learning (HRL), is thought to reflect a gradual transfer of serial-order information held in short-term memory to a longer-lasting representation of the sequence in long-term memory.

It has been hypothesized that this HRL provides a source of evidence for the role of implicit sequence memory mechanisms in language learning, particularly with respect to the long-term acquisition of novel phonological word-forms (Majerus \& Boukebza, 2013a; Page \& Norris, 2008, 2009; Szmalec, Duyck, Vandierendonck, Mata, \& Page, 2009; Szmalec, Page, \& Duyck, 2012). During novel word-form acquisition, learners need to segment word units from phonological (speech) input, either by tracking transitional probabilities among sounds in the incoming stream (Saffran, 2001; Saffran et al., 1996), or (alternatively) by the application of chunking mechanisms in memory (Thiessen \& Erickson, 2013). Regularly occurring sub-sequences gradually consolidate into long-term memory due to repeated exposure of their serially-ordered constituents (i.e., syllables/phonemes) (Page \& Norris, 2008, 2009). Hebb repetition learning of, for instance, a sequence of consonant-vowel syllables (e.g., lo-fo-du) is therefore, by hypothesis, functionally equivalent to the long-term memorization (hence, lexicalization) of a corresponding novel word-form (lofodu). In Page and Norris $(2008,2009)$, this learning is explained within a connectionist model that involves several layers of localist units representing phonemes or syllables, together with a learning mechanism that progressively consolidates a short and repeatedly presented sequence into one (localist) chunk representation in long-term memory. Experimental work has supported this idea by showing that subsequences acquired through HRL are represented in the mental lexicon just like novel words, 
exhibiting competition effects with existing words (which is an important indication of lexicalization, see Leach \& Samuel, 2007; Szmalec, Duyck, Vandierendonck, Mata, \& Page, 2009; Szmalec, Page, \& Duyck, 2012). HRL is also fast and long-lasting (Page, Cumming, Norris, McNeil, \& Hitch, 2013), and correlated with measures of word-learning and vocabulary knowledge (Majerus \& Boukebza, 2013b; Majerus, Poncelet, Elsen, \& Van der Linden, 2006); it is observed in children (Mosse \& Jarrold, 2008; Smalle et al., 2016); and it has been shown to be impaired in people with certain language disorders (Archibald \& Joanisse, 2013; Bogaerts, Szmalec, De Maeyer, Page, \& Duyck, 2016; Bogaerts, Szmalec, Hachmann, Page, \& Duyck, 2015; Hsu \& Bishop, 2014; Szmalec, Loncke, Page, \& Duyck, 2011).

Learning in the Hebb repetition task is further considered to depend on implicit learning mechanisms (though explicit awareness often naturally evolves across the repetitions) (Guerard, Saint-Aubin, Boucher, \& Tremblay, 2011). For instance, neither explicit awareness of the repeating sequence, nor explicit reproduction of the sequence (i.e., overt recall) during the recall phase, nor focal hippocampal lesions, seem necessarily to affect learning (Couture \& Tremblay, 2006; Gagnon, Bedard, \& Turcotte, 2005; Gagnon, Foster, Turcotte, \& Jongenelis, 2004; Guerard et al., 2011; Kalm \& Norris, 2016).

In the present study, participants were made explicitly aware of only one of two Hebb sequences (i.e., the sequences and their co-presented pictures) by the use of intentional encoding instructions issued prior to training (Gagnon et al., 2005), as well as by use of explicit cuing during the task (similar to Nemeth, Janacsek, \& Fiser, 2013). The other Hebb sequence remained implicit, in the sense that its presence was unannounced to participants. On the first day, two learning sessions were carried out, separated by a four-hour delay. One week and, then again, one year after learning, the sequences were re-presented to measure their long-term retention (cf. Bishop et al., 2012). Based on the previous observation of better delayed memory for nonwords in children than in adults, we expected to see better retention of the Hebb sequences in children than in adults, up to a one-year delay. Additionally, in line with previous findings that showed a decline in implicit learning, but not in explicit learning, after age twelve (Janacsek et al., 2012; Nemeth, Janacsek, \& Fiser, 2013), we 
expected to see child advantages particularly for the implicit (unannounced) Hebb sequence but not, or less so, for the explicit (instructed) Hebb sequence at which adults are hypothesized to perform well. Moreover, there is a reason to expect that the child advantage in the implicit condition should itself depend on the degree to which the children and the adults notice the unannounced repetition, and therefore switch to more explicit learning. That is, the age-effect should be reduced for participants who are more aware of the repetition. Correlations with post-learning awareness of the unannounced Hebb sequence, vocabulary knowledge and short-term memory capacity were additionally investigated to explore the potential role of explicit attention processes, lexical knowledge and short-term memory capacity in Hebb repetition learning (HRL) in both age groups.

\section{Methods}

\section{Participants}

Fifty-five participants took part in our longitudinal study, 29 children and 26 adults (Table 1). Children were recruited from two different schools in and around Ghent (Belgium). Adults were recruited by means of online advertising. All participants were Dutch speaking and had no reported history of speech, language, learning or hearing difficulties. All participants gave informed consent and received a financial compensation (€20) for their participation. Parental consent was obtained for the children's participation. The study was approved by the Psychology Ethics Commission at the Université catholique de Louvain.

\section{Materials}

Sequences of 9 syllables were presented to the participants for immediate serial recall. All syllables had a consonant-vowel structure (CV). The length of the sequences was matched to an average adult digit-span level, with two more items added to avoid ceiling effects. The same length was used for both the child and adult groups so that potential differences between the groups could not be attributed to differential input. To create the stimulus-sequences, four different sets (A to D), each set consisting of three CV-pools $(1,2,3)$ with nine different CVs in each CV-pool (see Table 2), were generated using WordGen (Duyck, Desmet, \& Verbeke, 2004). We confirmed that the summed 
bigram frequencies (as measured within the speaker's native language; Duyck et al., 2004) for the entire sequences were comparable within and across sets (i.e., all $p s>.05$ ). Each CV-pool within a set could be used either to generate filler sequences, or, directly, as one of the two Hebb sequences. This was counterbalanced across participants in a Latin square order (i.e., sequences 123, 231, 312 were used for Fillers, Hebb 1 and Hebb 2 respectively for participants 1, 2 and 3), so that Hebb learning could not be confounded by unknown particularities in the sequences themselves. Every three subsequent participants were allocated to one of the four sets (A-D) in turn. The allocation of participants to sets was matched across child and adult groups.

To facilitate word-segmentation processes in the HRL task ${ }^{1}$, and simultaneously to make the task more attractive for the children and similar to natural word-learning (where meaning is provided), the sequences were presented together with drawings of aliens derived from the TOTimals stimulus set (Gupta, 2003; Schwartz \& Smith, 1997). For each sequence of nine syllables, a given drawing was presented together with each subgroup of three successive syllables within that sequence (Figure 1), so that each three-syllable subsequence potentially became associated with the simultaneously presented drawing. From a total of 36 different drawings, nine drawings were associated with each set of three CV-pools. Of these nine drawings, three drawings were associated with the pool used to generate the filler sequences; another three drawings were associated with the first Hebb sequence and the remaining three drawings were associated with the second Hebb sequence. In each case, the three drawings associated with a given type of sequence (Filler, Hebb 1, Hebb 2) were clearly distinguishable from each other and they were always presented together with the CV-sequence in the same order. The order of the CV-syllables within the Hebb sequences remained fixed throughout learning, so that, for the Hebb sequences, the mapping was constant between a given drawing and a given subsequence of three successive syllables. The order of the syllables within the filler sequences was determined randomly. For this reason, there was no systematic mapping, for the filler sequences, between CV-syllables and drawings.

\footnotetext{
${ }^{1}$ In previous Hebb learning studies, chunking processes were facilitated by using pauses between every three syllables (see, Szmalec et al., 2009).
} 


\section{Procedure}

The experiment started with a familiarization phase during which participants were exposed to the syllable materials. Each participant listened carefully to the CVs that were also presented visually on the screen. Participants were instructed to repeat the CVs out loud, and they were corrected by the experimenter if the CV was not pronounced well. This familiarization phase was repeated three times to ensure that participants clearly distinguished the different syllables. All CVs were recorded by a female professional diction/pronunciation teacher and presented auditorily at 60 $\mathrm{dB}$ using Bose QC 15 headphones.

On the first day of testing, two sessions of 36 sequence trials were presented for immediate serial recall: these were sessions A and B. The two sessions were separated by a four-hour break in which participants continued their regular daily activities. The two types of Hebb sequence were mixed within the same session and each one was repeated on every third trial, implying twelve repetitions for each of the two Hebb sequences. These were interspersed with a total of twelve filler sequences, resulting in 36 sequence trials per session. The syllables within a sequence trial were presented auditorily, one at a time, each having a duration of $500 \mathrm{~ms}$ and a silent inter-stimulus interval of 500ms. The Hebb learning procedure was similar to that of previous studies (e.g., Szmalec et al., 2009; Smalle et al., 2016). Differently from previous studies, we accompanied each subsequence of three successive syllables within a given sequence with the visual presentation of a picture of an alien. The participants were told that they would see "aliens from the fictional planet Erinominus", presented simultaneously with auditory sequences of syllables. Each drawing appeared on the screen for $3 \mathrm{~s}$, and thus matched the time during which the three corresponding syllables were heard by the participant. The task was to repeat the syllables in the same order as presented, after all nine syllables had been heard.

Before learning started, the participants were also explicitly told that one sequence of nine speech sounds and the associated aliens were always repeated in the same order during the task. This is referred to as the explicit Hebb sequence or Hexplicit in the remainder of the paper. Every time 
Hexplicit was repeated, it was cued with a beeping tone and a red border around the screen.

Participants were not made aware of the occurrence of the second repeating sequence, from now on referred to as the implicit Hebb sequence or Himplicit. Two filler sequences were presented to familiarize participants with the immediate-serial-recall procedure. Learning started with presentation of a filler sequence followed by one of the two types of Hebb sequences (implicit or explicit), the other type of Hebb sequence (explicit or implicit), again a filler sequence, etc. In total, 12 filler sequences and 12 repetitions of the two types of Hebb sequences (i.e., 36 trials in total) were presented. The order of the two types of Hebb sequence was counterbalanced across participants. Immediately after presentation of each sequence, a recall screen was presented with the first alien and a question mark signaling that the participant had to repeat the first three syllables of the sequence. Subsequently (induced by a manual press on the spacebar), the next alien appeared with a question mark signaling the requirement to recall the next three syllables of the sequence, and so on, until all nine CVs of the sequence were recalled. Participants were allowed to say "blank" when they had forgotten a syllable at a particular serial position. The uttered syllables were recorded and simultaneously written down by the experimenter. For a syllable to be judged correct, the consonant and vowel in the heard syllable had to be present in the response, in the correct order, and without the insertion of additional phonemes. Consonants and vowels that were pronounced ambiguously (e.g. /p/ for /b/, or /e:/ for /i:/) were always scored in favor of the presented phoneme provided that the ambiguous sound (i.e., /p/ or /e:/) did not occur elsewhere in the sequence.

One week and, then again, one year after learning (see also Table 1), participants returned to the lab for re-testing on the HRL task in sessions C and D. The same Hebb procedure was used as above, but without a practice session. Participants were presented with 12 repetitions (for Hebb sequences) or presentations (for fillers) of each sequence type in session C (i.e., 36 trials in total), and 2 more sequence repetitions in session D (i.e. 6 trials in total). Across all four sessions, 114 trials were presented, including 38 fillers, 38 explicit Hebb repetitions, and 38 implicit Hebb repetitions.

At the end of each of the first three sessions (i.e., A, B, and C), a verbal awareness report assessing explicit knowledge of the repeating sequences was administered. This questionnaire 
included three increasingly specific questions (based on Gagnon et al., 2005; Janacsek et al., 2012; Turcotte, Gagnon, \& Poirier, 2005). In a first question, the experimenter asked whether participants were aware of the repeating Hebb sequence that followed the beep sound, presented within a red border. This was asked to ensure that the participant had understood the explicit instructions (this was the case for all participants). In a second question, the experimenter asked whether they noticed something else special in the task. Participants were free to respond. If the participant answered (or gave the impression) that there was another repetition, the experimenter acknowledged the participant as being aware. After this, two sub-questions were presented to all participants (also the unaware participants) 1. To rate confidence (about what they had noticed) on a scale from 1 'Not certain at all' to 10 ' $100 \%$ certain' and $\mathbf{2}$. To determine when in the task they started noticing something (i.e., at the beginning, middle or at the end of the task). Confidence ratings after each session were used as measure of post-learning awareness throughout the remainder of the paper. Participants who did not notice anything special, or noticed something irrelevant on the second question, automatically received the number zero on the confidence scale (and their answers on the sub-questions were ignored) so that no exclusion was needed in the analyses. The use of verbal reports after learning, in particular confidence ratings, is a common procedure and a relatively sensitive measure for demarcating explicit memory in humans (Cleeremans, Destrebecqz, \& Boyer, 1998; Sandberg, Timmermans, Overgaard, \& Cleeremans, 2010).

Finally, we measured verbal short-term memory capacity using the forward digit-span subscale of the Wechsler Intelligence Scales for Children-Revised (WISC-R) (Wechsler, 1974) following session B, and vocabulary knowledge using the Peabody Picture Vocabulary Test $\left(3^{\text {rd }}\right.$ Edition, PPVT-III) (Dunn \& Dunn, 1981), which was administered at the end of the experiment. Missing values were replaced by group means (Table 1). In addition, immediately at the end of learning in session $\mathrm{C}$, the twelve alien pictures that were presented during the Hebb task were randomly presented on the screen. Participants were asked whether they could recall the names of the aliens they had seen during the experiment. This naming task had no a priori theoretical motivation 
and was added primarily to make the procedure more game-like and thus attractive for the participating children.

\section{Statistical analyses}

We scored trial performance using McKelvie's scoring method (McKelvie, 1987). In a first step, the number of items that were in the correct position from left to right, up to the first error, was counted. Secondly, the same step was repeated from right to left, again up to the first error. After this, the number of items in any correct sequence of two or more items between the first error from the left and the first error from the right was counted. Finally, any other items that occurred in the correct position from left to right were counted. The maximum possible score using this method was nine. All trial scores were transformed into percentage scores. The trial scores at each session are plotted in Figure 2 (upper panel). Initial learning gains were determined by comparing the average scores over the first four trials with the average scores over the last four trials of session A (Turcotte et al., 2005) ${ }^{2}$. This comparison is plotted in Figure 2 (lower panel). Offline consolidation was determined by fitting within-session data with a power-law function model for both groups (Pan \& Rickard, 2015; Rickard, 2007). Delayed retention was then tested by comparing trial performance in the subsequent session (i.e., session B, or C, or D) with the performance level predicted by an extrapolation of the performance in the previous session (i.e., session A, or B, or C, respectively) (see also Adi-Japha et al., 2014 who used a similar approach). Extrapolation using power-law fitting is argued to be a more reliable measure for offline consolidation effects compared with measures solely based on pre-post difference scores (Pan and Rickard, 2015). All significant results are reported together with the $\eta_{\mathrm{p}}^{2}$ effect size and Greenhouse Geisser (GG) correction factors where applicable. Planned comparisons were conducted by Fisher's LSD comparisons.

\section{Results}

\section{Initial Hebb learning}

\footnotetext{
${ }^{2}$ Averaging across trials has been used for analyzing Hebb learning data in developmental samples (Mosse \& Jarrold, 2008; Archibald \& Joanisse, 2013; Smalle et al., 2016). It is, likewise, a procedure used in motor sequence learning (e.g. Adi-Japha et al., 2014; Csabi, Varszegi-Schulz, Janacsek, Malecek, \& Nemeth, 2014).
} 
The average performance levels for the initial and for the final four trials of session A were entered into a mixed-measures analysis of variance with Age group (child vs. adult) as betweensubjects factor, and Time point (initial vs. final) and Sequence type (Filler vs. Himplicit vs. Hexplicit) as repeated measures. While a main effect of Sequence type in favor of the Hebb sequence might provide some evidence of learning that sequence, only the demonstration of improvements in performance for repeated Hebb sequences relative to improvements in the baseline filler sequences may be considered a pure indication of learning. Thus, an interaction between Sequence type and Time point, due to higher scores on the Hebb sequences for the final time point, would provide evidence for Hebb learning (Smalle et al., 2016). Overall, adults showed higher recall scores than children (main effect of Age group, $F(1,53)=32.1, p<.001, \eta_{\mathrm{p}}^{2}=.38$ ). There was also a significant main effect of Time point, $F(1,53)=95.4, p<.001, \eta_{\mathrm{p}}^{2}=.64$, such that recall scores for the final time point were higher than recall scores for the initial time point, and a significant main effect of Sequence type, $F(2,106)=51.5, p<.001, \eta_{\mathrm{p}}^{2}=.49$. Comparisons revealed higher recall scores for both types of Hebb sequence compared with the filler sequence (Filler vs. Hexplicit, $F(1,53)=103.4$, $p<.001, \eta_{\mathrm{p}}^{2}=.66$, and Filler vs. Himplicit, $\left.F(1,53)=73.3, p<.001, \eta_{\mathrm{p}}^{2}=.58\right)$. There were no significant differences between the two types of Hebb sequence, $F(1,53)=2.04, p>.15, \eta_{\mathrm{p}}^{2}=.037$. Furthermore, there was a significant interaction between Time point and Sequence Type, $F(2,106)=$ 41.3, $p<.001, \eta_{\mathrm{p}}^{2}=.44$. Planned comparisons of the two-way interaction revealed a significant improvement across time points for Hexplicit, $F(1,53)=90.83, p<.001, \eta_{\mathrm{p}}^{2}=.63$, and Himplicit, $F(1,53)=73.5, p<.001, \eta_{\mathrm{p}}^{2}=.58$, but not for the filler sequence, $F<1$. There were no interactions with Age group, $F<1$. Overall, these results show that there was significant Hebb learning within session A, but its extent was not different for children and adults.

\section{Offline consolidation}

Based on previous observations in language and skill learning studies (Ashtamker \& Karni, 2013; Bishop et al., 2012), we had anticipated that children would retain the sequences better between sessions. To estimate delayed retention differences, a power-law function was fitted to the data of the 
initially trained Hebb sequences, and we tested for the possibility of additional improvements or decrements beyond what could have been expected from an extrapolation.

\section{Across four hours}

A power law function, $f(\mathrm{r})=\operatorname{ar}^{\mathrm{b}}$ where $r$ is the repetition number of the Hebb sequence and $a$ and $b$ are parameters estimated from the data, was fitted to the average recall data of session A for both the implicit and explicit Hebb sequence. This yielded a significant fit for the two age groups for both Hexplicit $\left(R^{2}>.96 ; p<.001\right)$ and Himplicit $\left(R^{2}>.93 ; p<.001\right)$. The exponential coefficients (bs) were significantly different from zero $(t \mathrm{~s}>14.69, p<.001$ for Hexplicit; and $t \mathrm{~s}>10.19, p<.001$ for Himplicit), indicating significant learning on session A for both sequences in both groups. The power-law models for the group-averaged data for the two types of Hebb sequence were extrapolated to 4 additional trial points and the differences between this extrapolation and each individual's performance at the four-hour post learning session (i.e., session B) were assessed (trial by trial) (Figure 3). Difference scores (that can be thought of as retention scores, or even retention and consolidation scores if significantly positive) were averaged across the four trials and compared between groups. For Himplicit, this revealed a main effect of Age group, $F(1,53)=5.7, p<.05, \eta_{\mathrm{p}}^{2}=$ .10 with children showing overall larger gains across the delay $(M$ difference score $=8.7 \%, \mathrm{SE}=4.1$, $t(28)=2.1, p<.05)$, in contrast with adults who showed numerical but non-significant decrements across the delay $(M$ difference score $=-6.5 \%, \mathrm{SE}=4.9, t(25)=-1.3, p=.196)$. In a post-hoc analysis, to control for unavoidably differing levels of overall recall across groups, we compared children and adults who showed similar recall scores on the last trial of session A. The $75 \%$ of children that showed the highest recall scores on trial 12 of session A $(\mathrm{n}=21 ; M$ trial score $=60.3 \%, \mathrm{SD}=21.3)$ were compared with the $75 \%$ of the adults that showed the lowest recall scores on this trial $(\mathrm{n}=19 ; M$ trial score $=61.4 \%, \mathrm{SD}=25.5$ ), these groups having closely matched levels of performance. In terms of the difference scores across sessions, the remaining groups still diverged, with the child group having a mean difference score of $13.7 \%(\mathrm{SD}=20.5), t(20)=3.06, p=.006$, and the adult group having a mean difference score of $-14.5 \%(\mathrm{SD}=21.9), t(18)=-2.36, p=.030$. Thus, although both these subgroups were at equivalent levels of performance at the end of session A, the child group still 
increased their performance (i.e., they retained and perhaps consolidated their sequence knowledge), while performance of the adult group dropped during the delay.

Overall, there was no clear age-effect for Hexplicit, $F(1,53)=2.5, p=.12$. Both groups showed retention although adults showed numerical but non-significant decrements $(M$ difference score for children $=2.1 \%, \mathrm{SE}=4.5, t(28)=.472, p=.640 ; M$ difference score for adults $=-8.1 \%, \mathrm{SE}$ $=4.7, \mathrm{t}(25)=-1.7, p=.097)$.

\section{Across one week}

A similar power-law function was fitted to the averaged recall data of session B for the two types of Hebb sequence. This yielded significant fits for the two age groups for both Hexplicit $\left(R^{2}>\right.$ $.88, \mathrm{p}<.001)$ and Himplicit $\left(R^{2}>.79, \mathrm{p}<.001\right)$. The exponential coefficients $(b \mathrm{~s})$ were significantly different from zero $(t \mathrm{~s}>8.18, \mathrm{p}<.001$, for Hexplicit, and $t \mathrm{~s}>5.91, \mathrm{p}<.001$ for Himplicit $)$, indicating significant learning within session B for both sequences for both groups. Again, the power-law models for the group-averaged data for the two types of Hebb sequence were extrapolated to four additional trials and the differences between this extrapolation and each individual's performance at the one-week post-learning session (i.e., Session C) were assessed trial-by-trial and averaged (Figure 4). For Himplicit, planned comparisons revealed a main effect of Age group, $F(1,53)=4.8, p=.033$, $\eta_{\mathrm{p}}^{2}=.08$, with children showing retention $(M$ difference score $=3.9 \%, \mathrm{SE}=4.3, t(28)=.89, p=.38)$, in contrast with adults who showed a significant decrement across the delay ( $M$ difference score $=$ $9.9 \%, \mathrm{SE}=4.5, t(25)=-2.21, p=.037)$. Again, when the $75 \%$ of the children that showed the highest score on trial 24 of session B $(n=21, M$ trial score $=83.1 \%, \mathrm{SD}=17.4)$ were compared with $75 \%$ of the adults that showed the lowest score on the end trial of session B $(n=19, M$ trial score $=87.7 \%$, $\mathrm{SD}=20.3)$, children retained, and even significantly increased performance across the delay $(M$ difference score $=12.0 \%, \mathrm{SD}=4.2, t(20)=2.85, p=.010)$ while adult performance decreased numerically though not reliably $(M$ difference score $=-11.01 \%, \mathrm{SD}=5.3, t(18)=-2.077, p=.052)$. 
There was no age-effect for Hexplicit, $F<1$, with both groups showing retention across the delay (child group, $M$ difference score $=-2.4 \%, \mathrm{SE}=4.3, t(28)=-.55, p=.59$; adult group, mean $=-$ $3.4 \%, \mathrm{SE}=3.3, t(25)=-1.01, p=.32)$.

\section{Across one year}

After one year, 28 children and 21 adults, i.e., $89 \%$ of the original sample, returned to the lab. Only these participants were included for extrapolation. A power law function, $f(r)=\operatorname{ar}^{\mathrm{b}}$, was fitted to the average trial scores of session $\mathrm{C}$ for the two types of Hebb sequences. This yielded significant fits for the two age groups for both Hexplicit $\left(R^{2}>.84, \mathrm{p}<.001\right)$ and Himplicit $\left(R^{2}>.66, \mathrm{p}<.01\right)$. The exponential coefficients $(b \mathrm{~s})$ were significantly different from zero $(t \mathrm{~s}>7.07, \mathrm{p}<.001$, for Hexplicit, and $t \mathrm{~s}>4.37, \mathrm{p}<.001$ for Himplicit), indicating significant learning within session $\mathrm{C}$ for both sequences for both groups. The power-law models for the group-averaged data for the two types of Hebb sequence were extrapolated to 2 additional trial points and the differences between this extrapolation and each individual's performance at the one-year-post-learning session (i.e., session D) was assessed (trial by trial) and averaged (Figure 5). For Himplicit, both groups' performance decreased across the delay (children, $M$ difference score $=-24.4 \%, \mathrm{SE}=3.6, t(27)=-6.82, p=.001$; adults, $M$ difference score $=-35.7 \%, \mathrm{SE}=5.9, t(20)=-5.97, p=.001)$. Adults decreased numerically, but not significantly, more than children (main effect of Age group, $F(1,47)=3.4, p=.07, \eta_{\mathrm{p}}^{2}=.067$; When the $75 \%$ of the children that showed the highest score on trial 36 of session C $(n=21, M$ trial score $=90.0 \%, \mathrm{SD}=14.4)$ were compared with the $75 \%$ of the adults that showed the lowest score on the end trial of session $\mathrm{C}(n=16, M$ trial score $=90.3 \%, \mathrm{SD}=16.6)$, adult performance dropped significantly more than that of children (Adults, $M$ difference score $=-46.6 \%, \mathrm{SD}=21.3$; Children, $M$ difference score $=-20.0 \%, \mathrm{SD}=18.5, t(35)=3.91, p<.001)$.

There was no age-effect, $F<1$, for Hexplicit with both groups decreasing across the delay (adults, $M$ difference score $=-32.4 \%, \mathrm{SE}=5.9, t(20)=-5.4, p<.001$; children, $M$ difference score $=-$ $38.7 \%, \mathrm{SE}=5.1, t(27)=-7.63, p<.001)$.

\section{Explicit knowledge of the sequences}


After session A, only $41.4 \%$ of the participants in the child group had noticed that there was another repetition in addition to the explicit sequence, while $73.1 \%$ of adults were aware of this. The $\chi 2$-test revealed a significant difference across age groups $\left(\chi_{(1)}=5.6, p<.029\right)$. By the end of session B, only $48.3 \%$ of the children had noticed the unannounced repetition while $84.6 \%$ of the adults were aware of it $\left(\chi 2_{(1)}=8.01, p<.005\right)$. Finally, by the end of session $\mathrm{C}$, almost all participants had noticed the unannounced repetition (i.e., $79.3 \%$ in the child group and $92.3 \%$ in the adult group), and the groups no longer differed significantly $\left(\chi 2_{(1)}=1.86, p>.259\right)$. To further characterize age differences in explicit knowledge of the sequence repetition for these aware participants, we compared the time point at which the aware participants reported that they noticed the repetition and how certain they were about the repetition (on a rating scale of 1 to 10). Overall, by the end of session C, all aware children had noticed the repetition later (i.e., around the middle of the second session) in the task than aware adults (i.e., at the end of the first session), $t(45)=2.58, p<.013$ ), and they were also less confident about their report $(M=8.3, S D=1.8)$ than adults $(M=9.8, S D=.53), t(45)=$ 3.6, $p<.001$. So, overall adults had gained more explicit knowledge of the sequence and at a faster rate than children.

It is possible that the child-adult differences obtained for offline consolidation of the implicit Hebb sequence (see main results) were driven by the fact that adults had gained more explicit knowledge of the repetitive occurrence of the unannounced repeating sequence. Contrary to our expectation of an implicit-memory deficit for adults, this would imply an adult deficit in explicit memory for an unannounced repeating sequence that they had nonetheless noticed more than had the children. To investigate this, we added confidence ratings (as a measure of explicit knowledge or awareness of the sequence) as a moderator variable into the univariate analysis of variance for the effect of Age-group on the retention of the unannounced repeating sequence (See also Supplementary Materials 1 for additional analyses of this issue, using approximately matched subgroups of children and adults). A summary of the results is provided in Table 3. Overall, these results indicate superior delayed implicit memory for children across all offline periods, irrespective of their level of awareness. The analysis also indicated a reliable relationship between retention and the moderator 
Awareness so that greater awareness of the unannounced repetition led to generally better retention (an effect that was statistically reliable for the adults but not for the children, see Table 5-7 in the subsequent correlation analyses). Across the one-year-delay, post-learning awareness further positively affected the retention scores for adults, but not for children, which is indicated by the significant interaction between Age and Awareness. We further address this finding in the discussion.

\section{Correlation with cognitive measures}

We further aimed to find out whether explicit knowledge of the sequences (i.e., confidence rating at the end of each session with inclusion of all participants), vocabulary knowledge and verbal short-term memory capacity are related to HRL in both groups. Therefore, a Pearson correlation analysis was conducted on the magnitude of learning at the end of session A (Table 4). Magnitude of learning was defined as immediate recall on the final time point of the Hebb sequence, whilst controlling for the performance at the final time point for the filler sequence. In addition, we performed correlational analyses between our cognitive measures and the extrapolated difference scores across each delay (see Table 5-7). All correlations show that HRL and subsequent offline retention were correlated positively with post-learning awareness and digit span in adults, and with vocabulary scores in children.

\section{Alien-naming task}

A logistic regression was performed to determine the effects of Group and Sequence Type, and their interaction on explicit alien-naming ability (correct or incorrect) after one week. The three aliens that were presented together with the filler sequence were never named correctly, which is not surprising given the fact that the order of the nine syllables of the filler sequences changed on every trial. These null scores were excluded from analysis. The more relevant analysis on the remaining data revealed a significant main effect of Group, $\chi 2(1)=10.5, p=.001$ with adults showing overall better naming of the aliens than children. This effect disappeared when recall accuracy for the Hebb sequences at the end of learning (i.e., the final trial of session C) was entered as covariate in the model, $\chi 2(1)=.186, p=.66$. If we additionally look at naming abilities depending on the order of the 
alien within the sequence, we only observed a group effect for naming the last alien of the sequence, $\chi 2(1)=8.2, p=.003$ and not for naming the first two aliens, $\chi 2(1)=2.2, p=.13$ and $\chi 2(1)=1.05, p=$ .30 , respectively. This indicates that the apparent superior naming ability for adults is likely driven by differences in immediate serial memory capacity and thus in the ability to attend to all nine syllables in the sequence. This latter is also illustrated in the serial position curves that are provided in the Supplementary Materials 2.

\section{Discussion}

Child-adult differences in implicit (vs. explicit) skill learning have been a topic of debate in cognitive science for decades (see for instance, Amso \& Davidow, 2012; Meulemans \& Van der Linden, 1998; Thomas et al., 2004). Starting from the observation of a developmental shift from implicit sequence learning to explicit learning in SRT tasks (Janacsek et al., 2012; Nemeth, Janacsek, $\&$ Fiser, 2013), together with the observation of superior delayed memory for novel word-forms in children (Bishop et al., 2012), we conducted a four-session, one-year longitudinal study in which we compared a group of children and adults on Hebb repetition learning. The Hebb repetition task is a well-known (implicit) sequential analogue of word-form learning (Page \& Norris, 2008, 2009; Szmalec et al., 2009, 2012). The same sequences of nine syllables, notionally subdivided into three groups of three syllables and associated with three pictures of aliens, were presented for immediate recall to a mixed group of 8 to 9-year-old children and adults. There were two (Hebb) sequences that were repeated on every third trial, of one of which participants were made explicitly aware. Longterm retention of the two Hebb sequences was tested over offline periods of 4 hours, one week and one year. Three important findings were obtained: (1) Despite overall lower performance in immediate serial recall for children, the increase in performance for the repeated sequences relative to the filler sequences (i.e. the Hebb effect) was equivalent for both groups; (2) Children showed better delayed retention of the implicit Hebb sequences across all offline periods; (3) Adults gained more (and at a faster rate) explicit awareness of the unannounced repeating sequence, but this could not explain the child advantage for offline retention performance of that sequence. 
First of all, we found that, despite their lower memory capacity (number of items reproduced correctly in order), children were not disadvantaged in learning rate, but improved their performance as much across repetitions as did adults. The finding that children learn the Hebb sequences at the same rate despite their lower performance on filler sequences is an important finding. It shows that children compensate for lower memory storage capacity by other, probably more implicit mechanisms, which become evident in the Hebb learning task. In an earlier Hebb-learning study, where the length of the Hebb sequences was adjusted to the working memory span of children and adult groups, therefore making the age-groups comparable on initial level of performance, children even outperformed adults on Hebb-repetition learning (Smalle et al., 2016). This earlier finding and the present work together, strengthen the hypothesis that children outperform adults in their use of implicit sequence-learning mechanisms that are related to language (see also, for instance, Smalle, Muylle, Szmalec \& Ducyk, 2017).

Second, we found evidence that the Hebbian-learned phonological sequences were better retained by children in subsequent consolidation phases across delays ranging from four hours up to 12 months. This was shown by a method of power-fitting and extrapolation similar to developmental work by Adi-Japha et al. (2014). Even when, on an exploratory basis, we extrapolate to more than 4 trial scores (Figure 6), our data show a fairly consistent pattern with children showing a gradual and non-intermittent evolution in performance while adults show a pattern of forgetting and slow recovery after some delay. The pattern of superior delayed memory in children emerged more strongly for the implicit Hebb sequence. Interestingly, this pattern was not diluted, and even got stronger, when we compared better performing children with worse performing adults by matching groups on end-trial scores, thus comparing across participants with more similar memory capacities. This supports our hypothesis that an underlying maturational effect on implicit memory is likely explaining the observed child superiority for delayed memory of new phonological word-forms and their associated pictures that are learned through implicit Hebb-learning principles.

Third, we found that awareness (and digit span) was positively correlated with performance in adults but not in children. The correlation with both post-learning awareness and digit span suggests 
that adults use well-developed cognitive resources such as attention and working memory when learning novel information, while such mechanisms are less readily available for children. Hebblearning performance in children showed a consistently significant correlation with vocabulary scores. This observation is not new and has also been reported in the statistical-learning literature previously (see for instance, Evans, Saffran, \& Robe-Torres, 2009), consistent with the claim that a common implicit-learning mechanism is used for word-acquisition in everyday life. In previous memoryrelated work, correlations have been found between non-word repetition, immediate serial recall, and vocabulary knowledge (Gathercole, 2006; Gupta \& Tisdale, 2009b; Majerus, Poncelet, Elsen, \& Van der Linden, 2006). In these studies, it was argued that a serial-order mechanism in short-term memory supported vocabulary development by facilitating learning of the phonological forms of new words during the initial learning stage (Majerus \& Boukebza, 2013b). Additionally, a learner with a high vocabulary size (as measured with the Peabody test) was proposed to be expected to have greater phonological knowledge than a learner with a small vocabulary; this, in turn, was thought to facilitate novel word-form learning. Evidence for positive feedback loops comes from computational work (Gupta \& Tisdale, 2009a). Hence, we argue that the correlation between HRL and vocabulary knowledge indicates that children's implicit serial-order learning abilities support the acquisition of novel word-forms that characterizes a fundamental aspect of their general vocabulary development. Adults' word-learning skills presumably rely on other explicit learning mechanisms too.

Adults had, overall, gained more explicit knowledge of the sequences than children. This was revealed from the post-learning awareness questionnaire for which adults gained awareness at a faster rate and with more confidence than children. Importantly, this age-dependent shift in explicit knowledge of the sequence (potentially reflecting explicit learning mechanisms) could not explain why adults forgot more across the delays than children (Table 3). Our results are more likely to reflect a maturational effect for delayed implicit memory of novel linguistic representations. This is in contrast to the instructional hypothesis that has been put forward as an alternative explanation for maturational differences in language learning (Lichtman, 2016). According to this hypothesis, children are superior implicit learners of language structures compared with adults, not because of 
cognitive maturation, but rather because adults receive more explicit instruction than children in the natural environment.

The results in Table 3 indicate a reliable relationship between retention and the moderator variable Awareness. As the positive correlations with Awareness presented in Tables 5-7 make clear, greater awareness of the unannounced repetition led to generally better retention, an effect that was statistically reliable for adults but not for children. The moderating effect at the one-year delay suggests that the more aware participants (predominantly the adults) became, the less the retention performance between the groups differed. In other words, the biggest child-adult difference in retention was found for those who were less aware of the repetition, which shows that the locus of the effect is implicit learning, rather than a mere effect of awareness. This is consistent with our expectation of an implicit-memory difference between children and adults, and does not support any alternative explanation based on adults having noticed the unannounced repetition, which they then remembered explicitly and preferentially forgot. At the four-hour and one-week delays there was no reliable moderation by Awareness of the effect of Age. This was only statistically reliable in the oneyear delay condition. By this end stage of the experiment, the 21 returning adults had all reported to be aware of the unannounced repetition, at a confidence level of eight ( 1 adult), nine ( 4 adults) or a maximum of ten (16 adults). By contrast, there was much more variability in the awareness scores of children, with six children still reporting no awareness of the unannounced repetition. We would, therefore, not want to over-interpret the interaction with Awareness, and the lack thereof in the other two delays, because it is based on a set of adults, all of whom have become highly aware of, and highly confident about, the unannounced repetition. Importantly, once adults have noticed the unannounced repetition, their performance resembles that in the explicit condition more, as we would expect where they are not reliably disadvantaged and equally retain relative to children.

Finn and colleagues demonstrated that adults' superior cognitive abilities, such as explicit attention and effort, may interact in a competitive or cooperative way with different aspects of language learning (Finn, Lee, Kraus, \& Hudson Kam, 2014). In their study, cognitive effort or attention disrupted the learning of phonological categories of novel word-forms, whereas 
segmentation of the word-forms (by statistical cues) benefitted from attention. The complex interacting nature of explicit and implicit learning in adults is not new in the skill -and language learning literature (Brown \& Robertson, 2007; Foerde, Knowlton, \& Poldrack, 2006; Nemeth, Janacsek, Polner, \& Kovacs, 2013; Poldrack et al., 2001), and it offers an interesting framework for why adults are good at some (such as lexico-semantics) but not all (such as phonology or grammar) language-learning tasks (Newport et al., 2001; Finn et al., 2014). In the current study, we found that post-learning awareness positively affected adults' memory performance one year later (when all participants had become aware of the sequence, though children at a lower confidence level). Moreover, we found a consistent positive correlation between post-learning awareness and HRL and retention in the adult group. Adults presumably rely to a different degree on more mature declarative memory mechanisms (like attentional control or working memory) that might further help explicit properties inherent to word-learning such as word segmentation processes (Finn et al., 2014), and/or more deliberate long-term memorization of the word-forms and their associated meanings. The sequence-picture (aliens) pairings during HRL might have induced explicit associative processes in the word-learning task in adults, especially at the end of one year when the initial syllable sequences are eventually consolidated in novel form-meaning mappings in declarative memory (Graf Estes, Evans, Alibali, \& Saffran, 2007; Gupta \& Tisdale, 2009b; Ullman, 2015). Also, the additional (explicit) naming task for the aliens at the end of one week (after session C) showed no reliable childadult differences in retrieving the correct form that was associated with the alien, once differences in recall capacity at the last Hebb learning trial had been controlled for. The exact competing versus cooperating nature of explicit attention processes in adults' word-learning, and in other language learning tasks, needs to be addressed in future work.

\section{Conclusion}

We have provided empirical evidence, collected within a controlled setting, for the hypothesis that children have the potential to outperform adults in the long-term consolidation of phonological sequences into novel word-forms (and their associated pictures), through Hebb repetition learning. Our findings highlight a complex interaction between word-learning and other cognitive abilities such 
as attention, working memory and vocabulary knowledge, offering some new perspectives on how children's, compared to adults', memories may be differentially involved in certain aspects of language learning. Our hypothesis is that children outperform adults on a (perhaps) domain-general learning mechanism, namely, implicit serial-order learning, while adults shift to a more explicit learning mechanism that can mask this implicit-learning difference. Explicit learning could either compete with, or otherwise interact with, implicit-learning abilities on particular tasks. Finally, and perhaps most importantly, this study proposes a theoretical (Hebb-learning) framework that can be further developed to improve our understanding of skill-learning abilities, such as language learning, across life.

\section{References}

Adi-Japha, E., Badir, R., Dorfberger, S., \& Karni, A. (2014). A matter of time: rapid motor memory stabilization in childhood. Dev Sci, 17(3), 424-433. doi:10.1111/desc.12132

Amso, D., \& Davidow, J. (2012). The development of implicit learning from infancy to adulthood: item frequencies, relations, and cognitive flexibility. Dev Psychobiol, 54(6), 664-673. doi:10.1002/dev.20587

Ashtamker, L., \& Karni, A. (2013). Motor memory in childhood: early expression of consolidation phase gains. Neurobiol Learn Mem, 106, 26-30. doi:10.1016/j.nlm.2013.07.003

Bishop, D. V., Barry, J. G., \& Hardiman, M. J. (2012). Delayed retention of new word-forms is better in children than adults regardless of language ability: a factorial two-way study. PLoS One, 7(5), e37326. doi:10.1371/journal.pone.0037326

Brown, R. M., \& Robertson, E. M. (2007). Off-line processing: reciprocal interactions between declarative and procedural memories. $J$ Neurosci, 27(39), 10468-10475. doi:10.1523/JNEUROSCI.2799-07.2007

Cleeremans, A., Destrebecqz, A., \& Boyer, M. (1998). Implicit learning: news from the front. Trends Cogn Sci, 2(10), 406-416. doi:http://dx.doi.org/10.1016/S1364-6613(98)01232-7

Couture, M., \& Tremblay, S. (2006). Exploring the characteristics of the visuospatial hebb repetition effect. Mem Cognit, 34(8), 1720-1729. doi:10.3758/BF03195933 
Craik, F. I., \& Bialystok, E. (2006). Cognition through the lifespan: mechanisms of change. Trends Cogn Sci, 10(3), 131-138.

Csabi, E., Varszegi-Schulz, M., Janacsek, K., Malecek, N., \& Nemeth, D. (2014). The consolidation of implicit sequence memory in obstructive sleep apnea. PLoS One, 9(10), e109010. doi:10.1371/journal.pone.0109010

Dunn, L. M., \& Dunn, L. M. (1981). Peabody Picture Vocabulary Test: Circle Pines, MN: American Guidance Service.

Duyck, W., Desmet, T., \& Verbeke, L. P. C. (2004). WordGen: A tool for word selection and nnonword generation in Dutch, English, German and French. Behavior Research Methods, Instruments \& Computers, 36(3), 488-499.

Evans, J. L., Saffran, J. R., \& Robe-Torres, K. (2009). Statistical Learning in Children With Specific Language Impairment. Journal of speech, language, and hearing research : JSLHR, 52(2), 10.1044/1092-4388(2009/1007-0189). doi:10.1044/1092-4388(2009/07-0189)

Ferman, S., \& Karni, A. (2010). No childhood advantage in the acquisition of skill in using an artifical language rule. PLoS One, 5(10), e13648. doi:10.1371/journal.pone.0013648

Finn, A. S., Lee, T., Kraus, A., \& Hudson Kam, C. L. (2014). When It Hurts (and Helps) to Try: The Role of Effort in Language Learning. PLoS One, 9(7), e101806. doi:10.1371/journal.pone.0101806

Foerde, K., Knowlton, B. J., \& Poldrack, R. A. (2006). Modulation of competing memory systems by distraction. Proc Natl Acad Sci U S A, 103(31), 11778-11783. doi:10.1073/pnas.0602659103

Gagnon, S., Bedard, M. J., \& Turcotte, J. (2005). The effect of old age on supra-span learning of visuo-spatial sequences under incidental and intentional encoding instructions. Brain Cogn, 59(3), 225-235. doi:10.1016/j.bandc.2005.07.001

Gagnon, S., Foster, J., Turcotte, J., \& Jongenelis, S. (2004). Involvement of the hippocampus in implicit learning of supra-span sequences: The case of sj. Cogn Neuropsychol, 21(8), 867882. doi:10.1080/02643290342000609

Gathercole, S. E. (2006). Nonword repetition and word learning: The nature of the relationship. Applied Psycholinguistics, 27(04), 513-543. 
Graf Estes, K., Evans, J. L., Alibali, M. W., \& Saffran, J. R. (2007). Can infants map meaning to newly segmented words? Statistical segmentation and word learning. Psychol Sci, 18(3), 254260. doi:10.1111/j.1467-9280.2007.01885.x

Guerard, K., Saint-Aubin, J., Boucher, P., \& Tremblay, S. (2011). The role of awareness in anticipation and recall performance in the Hebb repetition paradigm: implications for sequence learning. Mem Cognit, 39(6), 1012-1022. doi:10.3758/s13421-011-0084-1

Gupta, P. (2003). Examining the relationship between word learning, nonword repetition, and immediate serial recall in adults. $Q \quad J$ Exp Psychol A, 56(7), 1213-1236. doi:10.1080/02724980343000071

Gupta, P., \& Tisdale, J. (2009a). Does phonological short-term memory causally determine vocabulary learning? Toward a computational resolution of the debate. Journal of Memory and Language, 61(4), 481-502.

Gupta, P., \& Tisdale, J. (2009b). Word learning, phonological short-term memory, phonotactic probability and long-term memory: towards an integrated framework. Philosophical Transactions of the Royal Society B: Biological Sciences, 364(1536), 3755-3771.

Hebb, D. O. (1961). Brain mechanisms and learning. In J. F. Delafresnaye (Ed.), Distinctive features of learning in the higher animal (pp. 37-46). New York: Oxford University Press.

Janacsek, K., Fiser, J., \& Nemeth, D. (2012). The best time to acquire new skills: age-related differences in implicit sequence learning across the human lifespan. Dev Sci, 15(4), 496-505. doi:10.1111/j.1467-7687.2012.01150.x

Johnson, J. S., \& Newport, E. L. (1989). Critical period effects in second language learning: the influence of maturational state on the acquisition of English as a second language. Cogn Psychol, 21(1), 60-99.

Kalm, K., \& Norris, D. (2016). Recall is not necessary for verbal sequence learning. Mem Cognit, 44(1), 104-113. doi:10.3758/s13421-015-0544-0

Lichtman, K. (2016). Age and learning environment: Are children implicit second language learners? J Child Lang, 43(03), 707-730. 
Majerus, S., \& Boukebza, C. (2013a). Short-term memory for serial order supports vocabulary development: New evidence from a novel word learning paradigm. J Exp Child Psychol, 116(4), 811-828. doi:http://dx.doi.org/10.1016/j.jecp.2013.07.014

Majerus, S., \& Boukebza, C. (2013b). Short-term memory for serial order supports vocabulary development: new evidence from a novel word learning paradigm. J Exp Child Psychol, 116(4), 811-828. doi:10.1016/j.jecp.2013.07.014

Majerus, S., Poncelet, M., Elsen, B., \& Van der Linden, M. (2006). Exploring the relationship between new word learning and short-term memory for serial order recall, item recall, and item recognition. European Journal of Cognitive Psychology, 18(6), 848-873.

McKelvie, S. J. (1987). Learning and Awareness in the Hebb Digits Task. The Journal of General Psychology, 114(1), 75-88. doi:10.1080/00221309.1987.9711057

Meulemans, T., \& Van der Linden, M. (1998). Implicit Sequence learning in Children. J Exp Child Psychol, 69, 199-221.

Murphy, K., McKone, E., \& Slee, J. (2003). Dissociations between implicit and explicit memory in children: The role of strategic processing and the knowledge base. J Exp Child Psychol, 84(2), 124-165. doi:10.1016/s0022-0965(03)00002-x

Nemeth, D., Janacsek, K., \& Fiser, J. (2013). Age-dependent and coordinated shift in performance between implicit and explicit skill learning. Front Comput Neurosci, 7, 147. doi:10.3389/fncom.2013.00147

Nemeth, D., Janacsek, K., Polner, B., \& Kovacs, Z. A. (2013). Boosting Human Learning by Hypnosis. Cereb Cortex, 23(4), 801-805. doi:10.1093/cercor/bhs068

Newport, E. L., Bavelier, D., \& Neville, H. J. (2001). Critical thinking about critical periods: Perspectives on a critical period for language acquisition. Language, brain and cognitive development: Essays in honor of Jacques Mehler, 481-502.

Page, M. P. A., \& Norris, D. (2008). Is there a common mechanism underlying word-form learning and the Hebb repetition effect? Experimental Data and a modelling framework. . In A. Thorn \& M. P. A. Page (Eds.), Interactions between short-term and long-term memory in the verbal domain. 
Page, M. P. A., \& Norris, D. (2009). A model linking immediate serial recall, the Hebb repetition effect and the learning of phonological word forms. Philos Trans R Soc Lond B Biol Sci, 364(1536), 3737-3753. doi:10.1098/rstb.2009.0173

Pan, S. C., \& Rickard, T. C. (2015). Sleep and motor learning: Is there room for consolidation? Psychol Bull, 141(4), 812-834. doi:10.1037/bul0000009

Perez, L. A., Peynircioglu, Z. F., \& Blaxton, T. A. (1998). Developmental differences in implicit and explicit memory performance. J Exp Child Psychol, 70, 167-185.

Poldrack, R. A., Clark, J., Pare-Blagoev, E. J., Shohamy, D., Creso Moyano, J., Myers, C., \& Gluck, M. A. (2001). Interactive memory systems in the human brain. Nature, 414(6863), 546-550.

Poldrack, R. A., \& Packard, M. G. (2003). Competition among multiple memory systems: converging evidence from animal and human brain studies. Neuropsychologia, 41, 245-251.

Rickard, T. C. (2007). Forgetting and learning potentiation: dual consequences of between-session delays in cognitive skill learning. J Exp Psychol Learn Mem Cogn, 33(2), 297-304. doi:10.1037/0278-7393.33.2.297

Robertson, E. M. (2012). New insights in human memory interference and consolidation. Curr Biol, 22(2), R66-71. doi:10.1016/j.cub.2011.11.051

Sandberg, K., Timmermans, B., Overgaard, M., \& Cleeremans, A. (2010). Measuring consciousness: is one measure better than the other? Conscious Cogn, 19(4), 1069-1078. doi:10.1016/j.concog.2009.12.013

Schwartz, B. L., \& Smith, S. M. (1997). The retrieval of related information influences on tip-of-thetongue states. Journal of Memory and Language, 36, 68-86.

Smalle, E. H., Bogaerts, L., Simonis, M., Duyck, W., Page, M. P., Edwards, M. G., \& Szmalec, A. (2016). Can Chunk Size Differences Explain Developmental Changes in Lexical Learning? Front Psychol, 6, 1925. doi:10.3389/fpsyg.2015.01925

Smalle, E. H., Muylle, M., Szmalec, A., \& Duyck, W. (2017). The different time course of phonotactic constraint learning in children and adults: Evidence from speech errors. Journal of Experimental Psychology: Learning, Memory, and Cognition. 
Squire, L. (1992). Declarative and Nondeclarative Memory: Multiple Brain Systems Supporting Learning and Memory. Cognitive Neuroscience, Journal of, 4(3), 232-243. doi:10.1162/jocn.1992.4.3.232

Szmalec, A., Duyck, W., Vandierendonck, A., Mata, A. B., \& Page, M. P. (2009). The Hebb repetition effect as a laboratory analogue of novel word learning. $Q J$ Exp Psychol (Hove), 62(3), 435-443. doi:10.1080/17470210802386375

Szmalec, A., Page, M. P. A., \& Duyck, W. (2012). The development of long-term lexical representations through Hebb repetition learning. Journal of Memory and Language, 67(3), 342-354. doi:10.1016/j.jml.2012.07.001

Thomas, K. M., Hunt, R. H., Vizueta, N., Sommer, T., Durston, S., Yang, Y., \& Worden, M. S. (2004). Evidence of developmental differences in implicit sequence learning: an fMRI study of chidren and adults. J Cogn Neurosci, 16(8), 1339-1351.

Turcotte, J., Gagnon, S., \& Poirier, M. (2005). The effect of old age on the learning of supraspan sequences. Psychol Aging, 20(2), 251-260. doi:10.1037/0882-7974.20.2.251

Ullman, M. T. (2004). Contributions of memory circuits to language: the declarative/ procedural model. Cognition, 92(1-2), 717-726.

Ullman, M. T. (2015). The declarative/procedural model: a neurobiological model of language learning, knowledge and use. Hickok, G., Small, SA (Eds.), The Neurobiology of Language. Elsevier, San Diego, CA.

Weber-Fox, C. M., \& Neville, H. J. (1996). Maturational constraints on functional specializations for language processing: ERP and behavioral evidence in bilingual speakers. $J$ Cogn Neurosci, $8(3), 231-256$.

Wechsler, D. (1974). Wechsler intelligence scale for children—revised. New York: Psychological corporation.

Wilhelm, I., Prehn-Kristensen, A., \& Born, J. (2012). Sleep-dependent memory consolidation--what can be learnt from children? Neurosci Biobehav Rev, 36(7), 1718-1728. doi:10.1016/j.neubiorev.2012.03.002 
Table 1. Gender, laterality, age, cognitive test scores and session intervals divided by age group.

\begin{tabular}{|c|c|c|c|}
\hline & $\begin{array}{c}\text { Children } \\
(\mathrm{n}=15 \mathrm{~F} / 14 \mathrm{M})\end{array}$ & $\begin{array}{c}\text { Adults } \\
(\mathrm{n}=17 \mathrm{~F} / 9 \mathrm{M})\end{array}$ & $\begin{array}{c}\text { Group } \\
\text { difference }\end{array}$ \\
\hline Age (yr) & $8.7(.47)$ & 35.5 (11.09) & $p<.001$ \\
\hline Digit Forward Span $(\max =9)$ & $5.8(1.5)$ & $7.3(1.7)$ & $p<.001$ \\
\hline $\begin{array}{l}\text { Peabody Vocabulary Test }-3^{\text {rd }} \text { ed. (PPVT-III), } \\
\max =204, r s\end{array}$ & $111^{(\mathrm{a})}(12)$ & $185^{(\mathrm{b})}(7)$ & $p<.001$ \\
\hline Session Interval & & & \\
\hline $\mathrm{A}-\mathrm{B}$ (in hours) & $4: 47(.03)$ & $4: 34(.03)$ & $n s$ \\
\hline $\mathrm{B}-\mathrm{C}$ (in days) & $7(1.1)$ & $7(1.0)$ & $n s$ \\
\hline $\mathrm{C}-\mathrm{D}$ (in months) & $11.9(.30)$ & $12.0(.47)$ & $n s$ \\
\hline
\end{tabular}

$\mathrm{F}$ = female; $\mathrm{M}$ = male; $\mathrm{rs}$ = raw score; (a) one missing value replaced by the mean; (b) four missing values replaced by the mean. 
Table 2. Stimulus materials for the Hebb learning task

\section{$\begin{array}{lllllllllll}\text { SET } & \text { CV-pool } & \text { CV1 } & \text { CV2 } & \text { CV3 } & \text { CV4 } & \text { CV5 } & \text { CV6 } & \text { CV7 } & \text { CV8 } & \text { CV9 }\end{array}$}

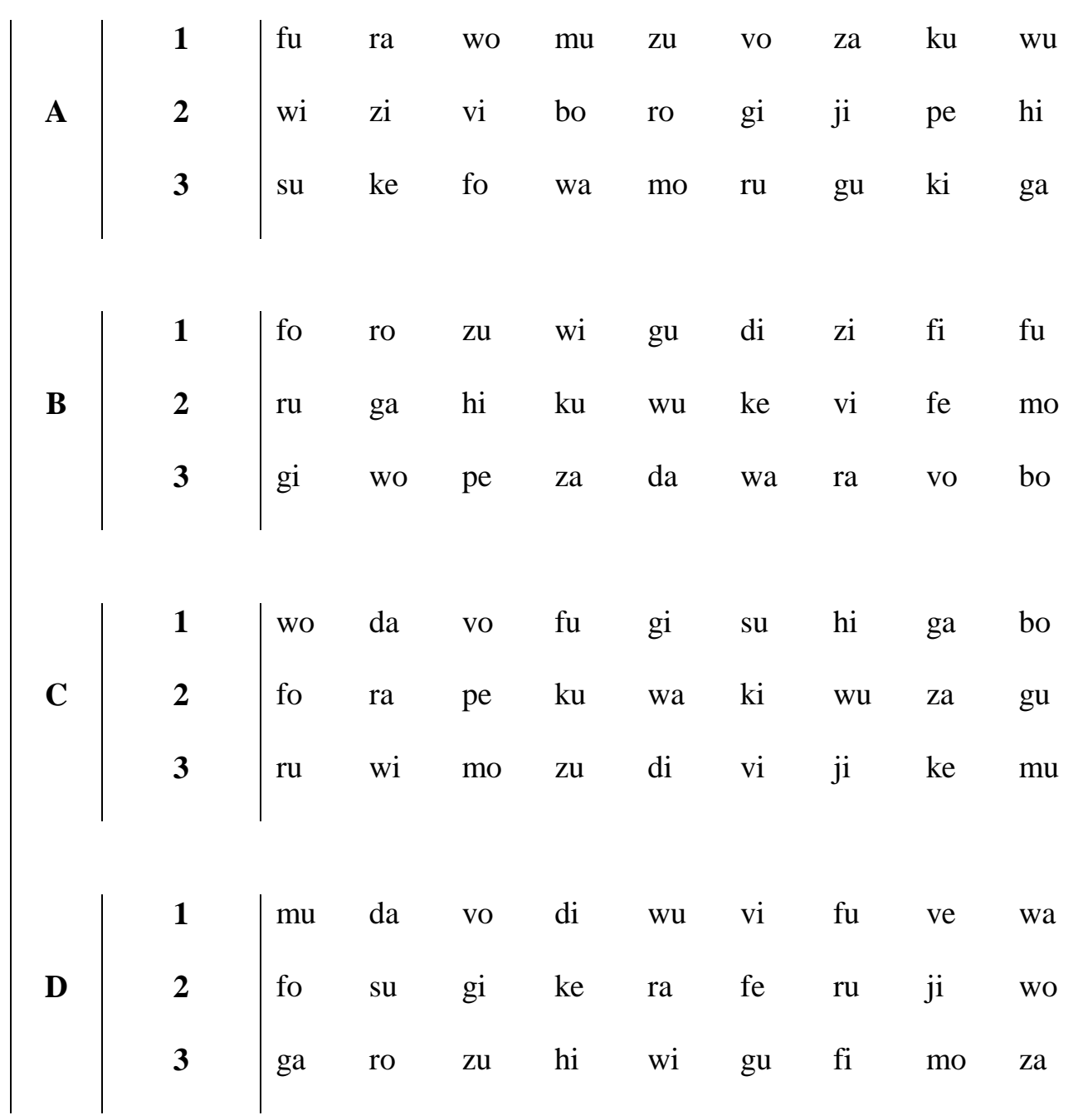


Table 3. Results of the moderation analysis, with retention (i.e., the averaged difference between the extrapolated and the observed scores) of implicitly presented material as the dependent variable, Age group as the factor, and Awareness (i.e., confidence rating) of the unannounced sequence entered as a potential moderator.

\begin{tabular}{|c|c|c|c|c|c|c|c|c|c|}
\hline \multirow[b]{2}{*}{ Variable } & \multicolumn{3}{|c|}{ Across four hours } & \multicolumn{3}{|c|}{ Across one week } & \multicolumn{3}{|c|}{ Across one year } \\
\hline & $F$ & Sig. & $n_{p}^{2}$ & $F$ & Sig. & $n_{p}^{2}$ & $F$ & Sig. & $n_{p}^{2}$ \\
\hline Age group & 9.59 & $.003^{* *}$ & .158 & 10.38 & $.002 * *$ & -.169 & 6.78 & $.012 *$ & .131 \\
\hline Awareness & 10.65 & $.002 * *$ & .173 & 11.33 & $.001 * *$ & .182 & 6.40 & $.015^{*}$ & .124 \\
\hline Age group*Awareness & .800 & .375 & .015 & 2.38 & .129 & .045 & 6.03 & $.018^{*}$ & .118 \\
\hline$R^{2}$ & & .263 & & & .256 & & & .176 & \\
\hline Adjusted $R^{2}$ & & .219 & & & .212 & & & .121 & \\
\hline
\end{tabular}


Table 4. Partial correlations between the magnitude of HRL (whilst controlling for filler performance) and post-learning awareness of the unannounced sequence (collected after session A), Peabody vocabulary score, and digit span. $D f$ equals 26 for children and 23 for adults.

\begin{tabular}{|c|c|c|c|c|}
\hline \multicolumn{5}{|c|}{ Implicit type of Hebb sequence } \\
\hline & \multicolumn{2}{|c|}{ Children } & \multicolumn{2}{|c|}{ Adults } \\
\hline & $r$ & Sig. & $r$ & Sig. \\
\hline Post-learning awareness & .244 & .211 & .525 & $.007 * *$ \\
\hline Peabody vocabulary score & .438 & $.020^{*}$ & .056 & .729 \\
\hline Digit Span & .089 & .651 & .294 & .154 \\
\hline \multicolumn{5}{|c|}{ Explicit type of Hebb sequence } \\
\hline & \multicolumn{2}{|c|}{ Children } & \multicolumn{2}{|c|}{ Adults } \\
\hline & $r$ & Sig. & $r$ & Sig. \\
\hline Peabody vocabulary score & .343 & .072 & -.085 & .687 \\
\hline Digit Span & .126 & .524 & -.261 & .208 \\
\hline
\end{tabular}


Table 5. Pearson correlations between average difference scores across four hours and post-learning awareness of the unannounced sequence (collected after session A), Peabody vocabulary score, and digit span. $N$ equals 29 for children and 26 for adults.

\begin{tabular}{lccccc}
\hline Implicit type of Hebb sequence & \multicolumn{2}{c}{ Children } & \multicolumn{2}{c}{ Adults } \\
\hline & $\boldsymbol{r}$ & Sig. & $\boldsymbol{r}$ & Sig. \\
\hline Post-learning awareness & .312 & .099 & .507 & $.008^{* *}$ \\
Peabody vocabulary score & .434 & $.019^{*}$ & .145 & .480 \\
Digit Span & .208 & .279 & .373 & $.067^{\circ}$ \\
\hline Explicit type of Hebb sequence & \multicolumn{3}{c}{ Children } & \multicolumn{2}{c}{ Adults } \\
\hline & \multicolumn{2}{c}{ Sig. } & \multicolumn{2}{c}{ Sig. } \\
\hline Peabody vocabulary score & .267 & .161 & .203 & .320 \\
Digit Span & -085 & .661 & .390 & $.054^{\circ}$ \\
\hline
\end{tabular}


Table 6. Pearson correlations between averaged difference scores across one week and post-learning awareness (collected after session B), Peabody vocabulary score, and digit span. $N$ equals 29 for children and 26 for adults.

\begin{tabular}{lccccc}
\hline Implicit type of Hebb sequence & \multicolumn{3}{c}{ Children } & \multicolumn{2}{c}{ Adults } \\
\hline & $\boldsymbol{r}$ & Sig. & $\boldsymbol{r}$ & Sig. \\
\hline Post-learning awareness & .255 & .181 & .581 & $.002^{* *}$ \\
Peabody vocabulary score & .402 & $.032^{*}$ & .028 & .891 \\
Digit Span & .092 & .634 & .266 & .277 \\
\hline Explicit type of Hebb sequence & \multicolumn{3}{c}{ Children } & \multicolumn{2}{c}{ Adults } \\
\hline & \multicolumn{2}{c}{$\boldsymbol{r}$} & \multicolumn{2}{c}{ Sig. } & \multicolumn{2}{c}{ Sig. } \\
\hline Peabody vocabulary score & .260 & .174 & .178 & .383 \\
Digit Span & -.014 & .944 & .443 & $.027^{*}$ \\
\hline
\end{tabular}


Table 7. Pearson correlations between averaged difference scores across one year and post-learning awareness (collected after session C), Peabody vocabulary score, and digit span. $N$ equals 28 for children and 21 for adults.

\begin{tabular}{|c|c|c|c|c|}
\hline \multicolumn{5}{|l|}{ Implicit type of Hebb sequence } \\
\hline & \multicolumn{2}{|c|}{ Children } & \multicolumn{2}{|c|}{ Adults } \\
\hline & $r$ & Sig. & $r$ & Sig. \\
\hline Post-learning awareness & .065 & .741 & .449 & $.040 *$ \\
\hline Peabody vocabulary score & .368 & $.054^{\circ}$ & .102 & .659 \\
\hline Digit Span & .332 & .084 & .442 & $.051^{\circ}$ \\
\hline \multicolumn{5}{|l|}{ Explicit type of Hebb sequence } \\
\hline & \multicolumn{2}{|c|}{ Children } & \multicolumn{2}{|c|}{ Adults } \\
\hline & $r$ & Sig. & $r$ & Sig. \\
\hline Peabody vocabulary score & .341 & .076 & .231 & .314 \\
\hline Digit Span & -.196 & .319 & .545 & $.01 * *$ \\
\hline
\end{tabular}


Figure 1. Design of the experiment. The Hebb Repetition Learning Task (HRL). For session A, B and C, 36 trials were presented for immediate serial recall (12 filler sequences and two Hebb sequences that were each repeated 12 times). In session D, six trials were presented (two filler sequences and two Hebb repetitions).

Figure 2. Performance (percentage of correct scores) as a function of Sequence Type (Filler, Explicit Hebb, Implicit Hebb) and Age group (Children and Adults). 1. Upper panel: performance across all sessions (i.e. repetition 1-12 for session A, repetition 13-24 for session B, repetition 25-36 for session C and repetition 1-2 for session D). 2. Lower panel: Performance across two time points (initial vs. final) in session A. Error bars denote SEM.

Figure 3. Offline consolidation performance after four hours as a function of Sequence Type (Explicit Hebb, Implicit Hebb) and Age group (Children and Adults). Hebb learning during session A is shown together with an extrapolation to four additional data points in session B. 1. Left panel: performance for the implicit Hebb sequence. 2. Right panel: performance for the explicit type of Hebb sequence. Error bars denote SEM.

Figure 4. Offline consolidation performance across one week as a function of Sequence Type (Explicit Hebb, Implicit Hebb) and Age group (Children and Adults). Hebb learning during session B is shown together with an extrapolation to four additional data points in session C. 1. Left panel: performance for the implicit Hebb sequence. 2. Right panel: performance for the explicit Hebb sequence. Error bars denote SEM.

Figure 5. Offline consolidation performance across one week as a function of Sequence Type (Explicit Hebb, Implicit Hebb) and Age group (Children and Adults). Hebb learning during session C is shown together with an extrapolation to two additional data points in session D. 1. Left panel: performance for the implicit Hebb sequence. 2. Right panel: performance for the explicit Hebb sequence. Error bars denote SEM. 
Figure 6. A visual depiction of learning performance for the implicit Hebb sequence in both children and adults, at each session, together with its power fit function and an exploratory extrapolation to its subsequent session. Error bars denote SEM. 
- Filler

Adults

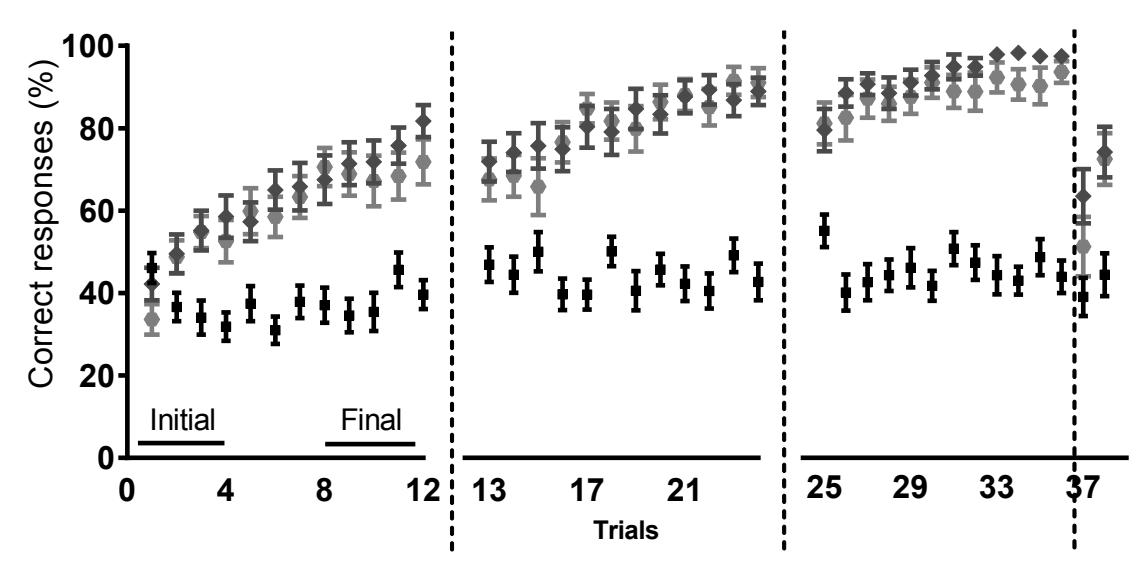

Session A

Session B

Session C

Session D
Children

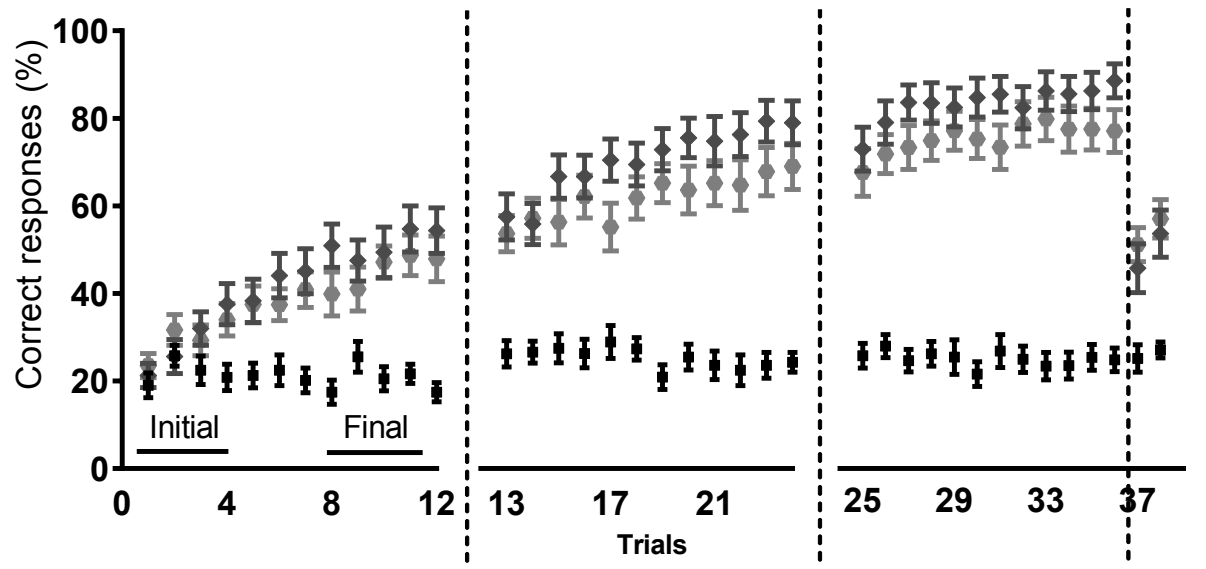

Session A

Session B

Session C

Session D
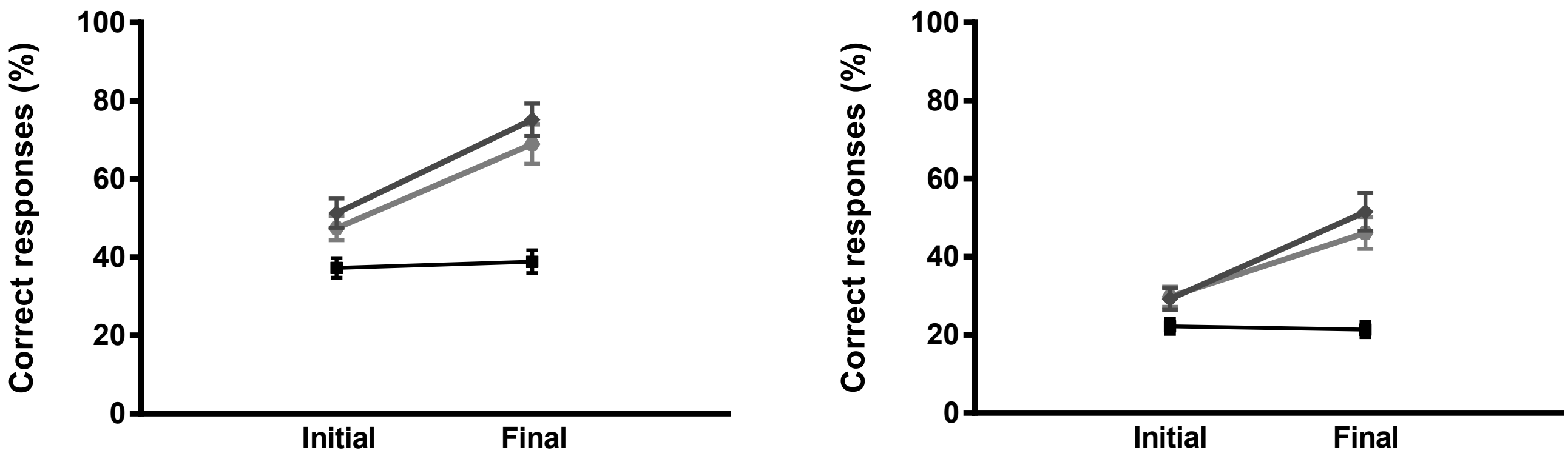
$\mp$ Hebb - Adults

$\nabla \quad$ Extrapolated - Adults

$\rightarrow$ Hebb - Children

$\Delta$ Extrapolated - Children

Implicit

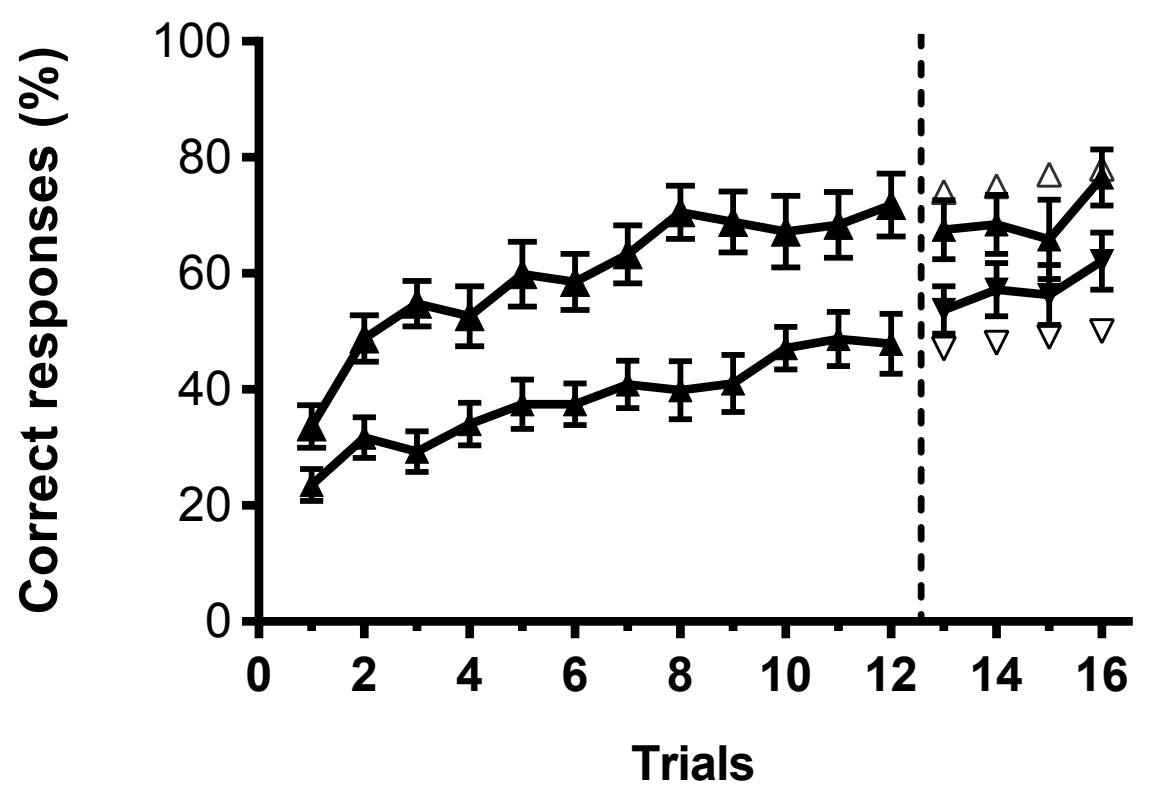

Explicit

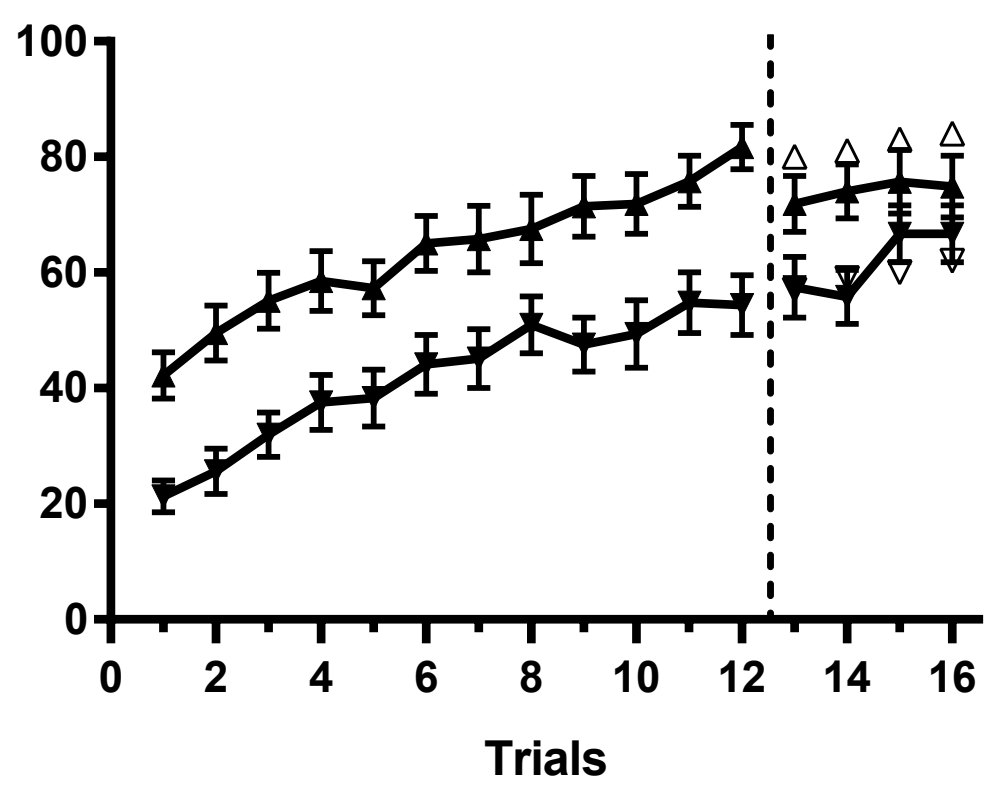


\pm Hebb - Adults

$\triangle$ Extrapolated - Adults

$\rightarrow$ Hebb - Children

$\nabla \quad$ Extraploated - children

Implicit

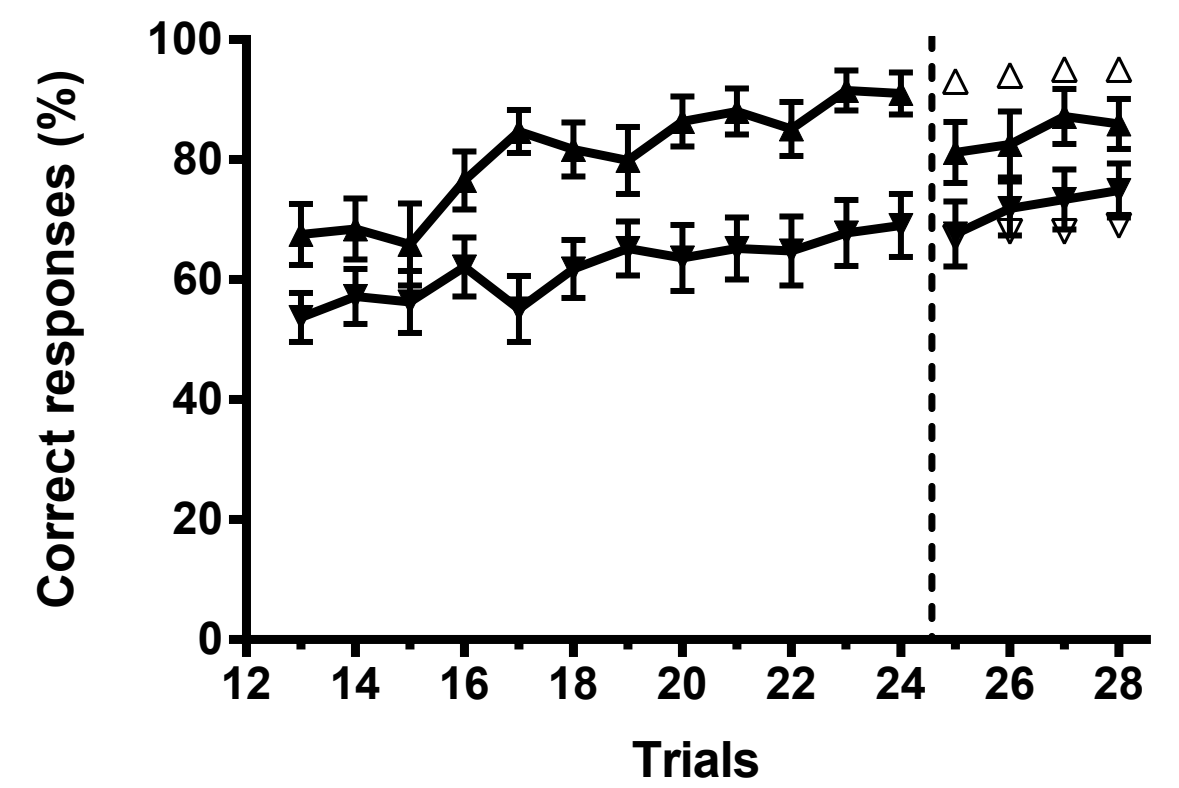

Explicit

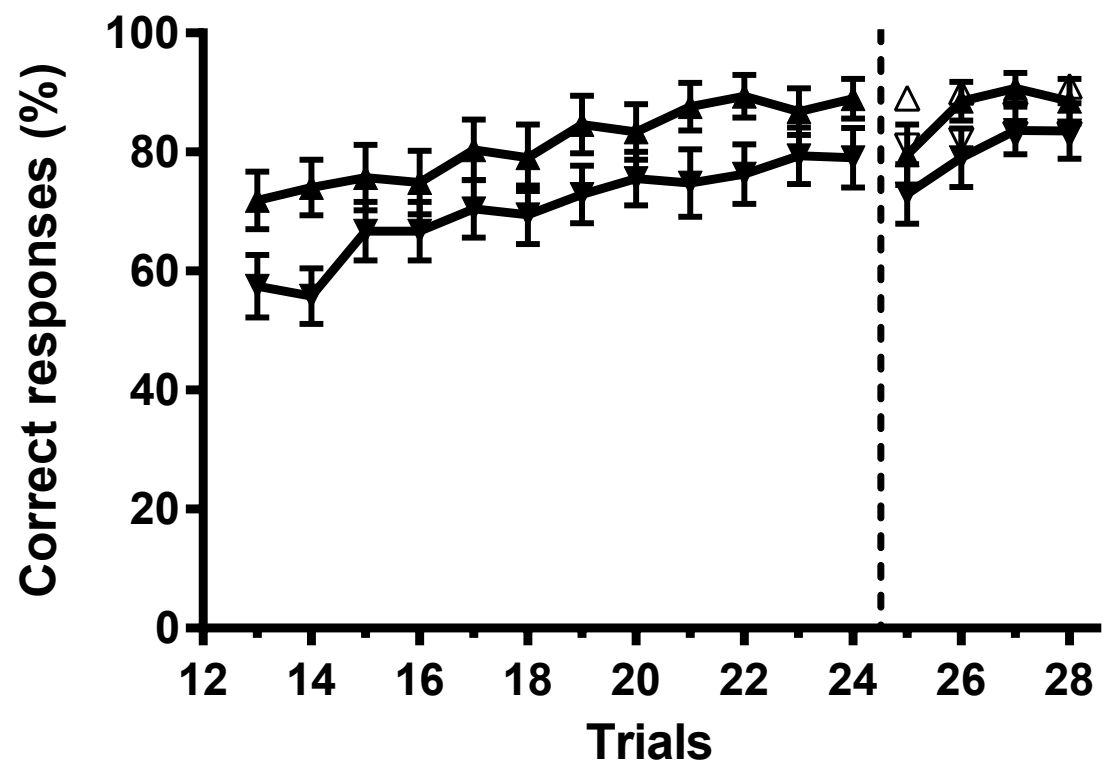


\pm Hebb- Adults

$\Delta$ Extraploated - Adults

$\rightarrow$ Hebb - Children

$\nabla$ Extrapolated- Children

\section{Implicit}

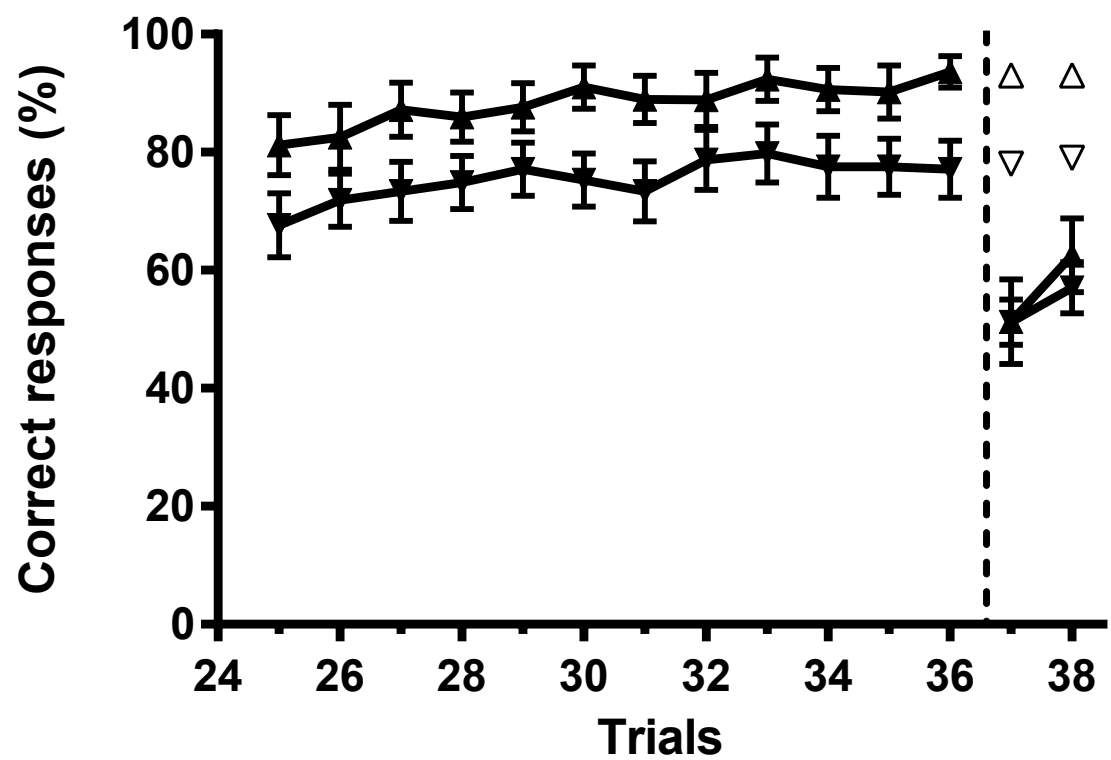

\section{Explicit}

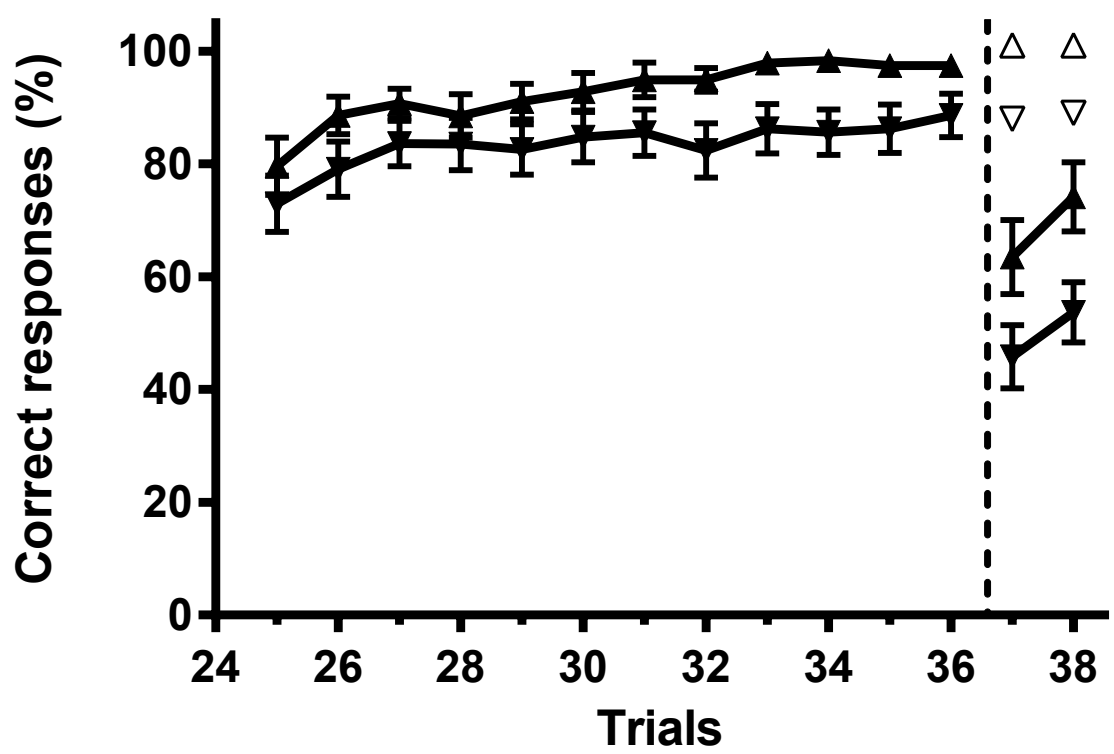




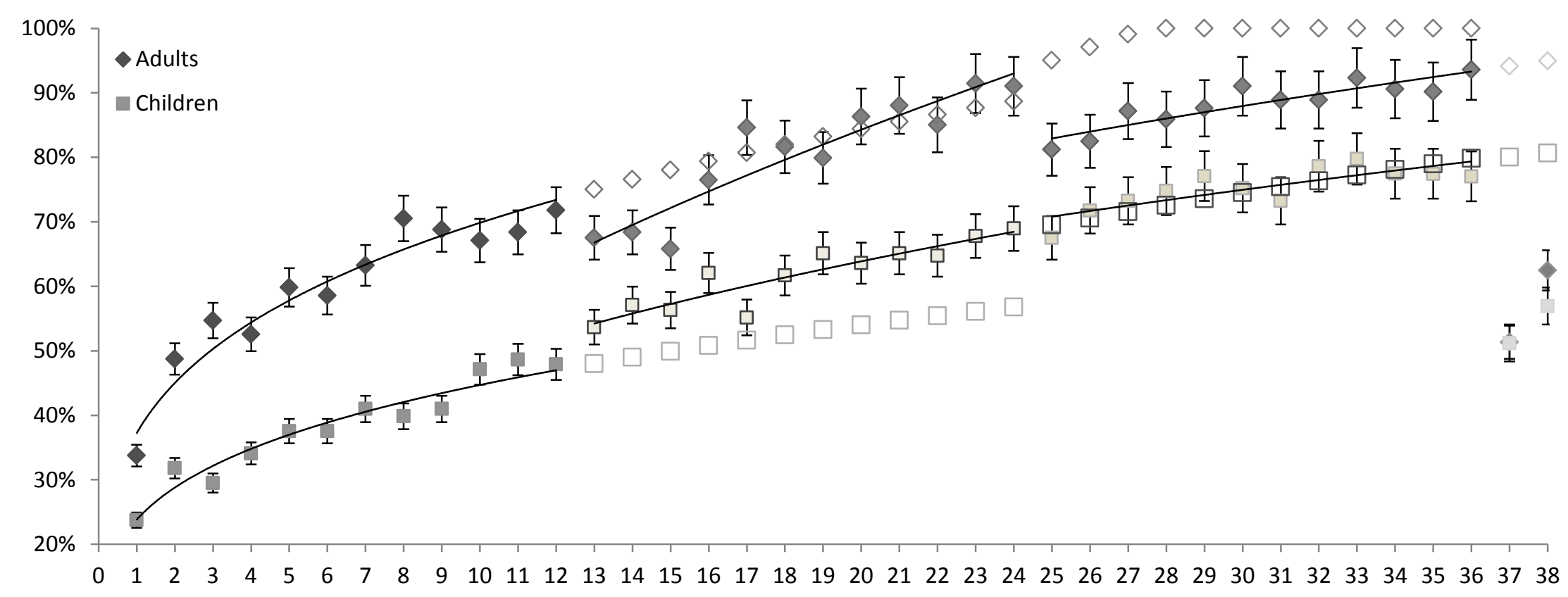




\section{Supplementary Materials 1}

Supporting information for the post-hoc analyses for offline consolidation:

It is possible that the child-adult differences obtained for offline consolidation of the implicit Hebb sequence (see main results) were driven by the fact that adults had gained more explicit knowledge of the repetitive occurrence of the hidden sequence. To investigate this, post-hoc analyses were conducted in which we compared difference scores between children and adults that showed an equivalent level of explicit knowledge (or awareness) of the hidden sequence (measured by their rate of confidence at the end of learning on the previous session).

Across four hours: The $75 \%$ of the children that reported the highest awareness (collected after session A, $\mathrm{n}=21, M$ confidence rating $=4.2, \mathrm{SD}=4.0$ ) were compared with the $75 \%$ of the adults that reported the lowest awareness $(\mathrm{n}=19, M$ confidence rating $=4.6, \mathrm{SD}=3.9)$ so that they were matched on level of explicit knowledge of the sequence (no group difference on rate of confidence, $t(38)=-0.354, p=.73$ ). In terms of difference scores, the two groups still diverged, $t(38)=-2.48, p=.018$, with children showing a significant gain of $10.3 \%(\mathrm{SD}=22.5), t(20)=2.1, p=.05$, and adults showing a non-significant decrement of $-8.8 \%(\mathrm{SD}=26.1), t(18)=-1.47, p=.16$.

Across one week: The $75 \%$ most aware children (collected after session $\mathrm{B}, \mathrm{n}=21, M$ certainty $=5.5, \mathrm{SD}$ $=4.3)$ and the $75 \%$ least aware adults $(\mathrm{n}=19, M$ certainty $=7.1, \mathrm{SD}=4.0, t(38)=-1.21, p=.24)$, again differed on the difference scores, $t(38)=2.91, p=.006$, with children showing retention of performance $(\mathrm{M}=6.3 \%, \mathrm{SD}=21.4, t(20)=2.1, p=.19)$ and adults showing a significant decrement of $-14.7 \%(\mathrm{SD}=$ 24.8), $t(18)=-2.6, p=.02$. 
Across one year: When the sixteen ${ }^{1}$ most aware children (reported after session $\mathrm{C}, M$ certainty $=9.4, \mathrm{SD}$ $=.72)$ were compared with the sixteen least aware adults $(n=16, M$ certainty $=9.7, \mathrm{SD}=.60, t(30)=-$ $1.33, p=.19$ ), adults still decreased more than children (Adults, $M$ difference score $=-46.6 \%, \mathrm{SD}=21.4$; Children, $M$ difference score $=-24.3 \%, \mathrm{SD}=19.5, t(30)=3.08, p=.004)$.

\section{Supplementary Materials 2}

Below, we provide the serial-position curves that result from the current data-set, for children (left) and adults (right), separately for all sessions (A, B and C).

In line with Mosse and Jarrold (2008), children showed reliable Hebb effects at early sequence positions but not yet at the end of the sequence at the start of learning (session A) - though the comparison here is somewhat compromised by a floor effect. In sessions B and C, a shift occurred towards all sequence positions but learning remains more pronounced for the initial items. Adults, in contrast, showed Hebb effects at all positions already during the first session (note that the comparison here is less compromised by a floor effect), though learning appeared here somewhat stronger for the initial items. The latter is in line with findings from Hitch, Fastame, and Flude (2005).

So it appears that Hebb learning exerts its effect primarily on the first three (maybe six) syllables for both groups, and moves gradually to the following syllables as learning proceeds. There also seems to be a different strategy-use for both groups, with children focusing more on the initial items retained in shortterm memory while adults more rapidly attend to all items. This difference in strategy is probably a consequence of differences in short-term memory capacity since the length of the sequences (nine syllables) surpasses memory span for children more than for adults.

\footnotetext{
${ }^{1}$ If we selected the $75 \%$ most aware children $(M$ certainty $=8.7, \mathrm{SD}=1.6)$, we were not able to match awareness reports of the children with those of the adults. Therefore we selected the 16 participants who were at the extreme in both groups.
} 

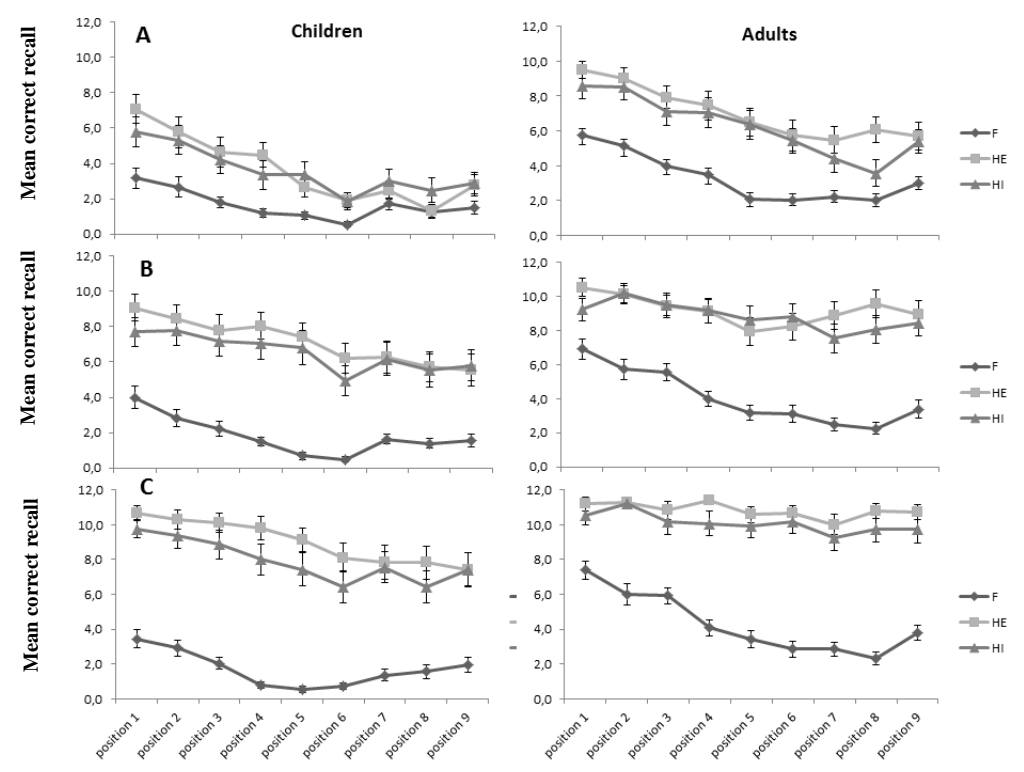

Figure 1. Mean correct recall as a function of serial position of syllables in the sequences (filler, Hebb implicit and Hebb explicit) in the Hebb learning paradigm, separately for all sessions (A, B and C). 